\title{
On the exact multiplicity of stable ground states of non-Lipschitz semilinear elliptic equations for some classes of starshaped sets
}

https://doi.org/10.1515/anona-2020-0030

Received January 30, 2019; accepted February 13, 2019.

Abstract: We prove the exact multiplicity of flat and compact support stable solutions of an autonomous nonLipschitz semilinear elliptic equation of eigenvalue type according to the dimension $\mathrm{N}$ and the two exponents, $0<\alpha<\beta<1$, of the involved nonlinearites. Suitable assumptions are made on the spatial domain $\Omega$ where the problem is formulated in order to avoid a possible continuum of those solutions and, on the contrary, to ensure the exact number of solutions according to the nature of the domain $\Omega$. Our results also clarify some previous works in the literature. The main techniques of proof are a Pohozhaev's type identity and some fibering type arguments in the variational approach.

Keywords: semilinear elliptic equation, non-Lipschitz terms, spectral problem, Pohozaev identity, flat and compact support ground states, sharp multiplicity

MSC: 35J60, 35J96, 35R35, 53C45

\section{Introduction}

In this paper we study the existence of non-negative solutions of the following problem

$$
\left\{\begin{array}{l}
-\Delta u+|u|^{\alpha-1} u=\lambda|u|^{\beta-1} u \text { in } \Omega, \\
u=0 \text { on } \partial \Omega
\end{array}\right.
$$

Here $\Omega$ is a bounded domain in $\mathbb{R}^{N}, N \geq 3$, with a smooth boundary $\partial \Omega$, which is strictly star-shaped with respect to a point $x_{0} \in \mathbb{R}^{N}$ (which will be identified as the origin of coordinates if no confusion may arise), $\lambda$ is a real parameter, $0<\alpha<\beta<1$. By a weak solution of $P(\alpha, \beta, \lambda)$ we mean a critical point $u \in H_{0}^{1}:=H_{0}^{1}(\Omega)$ of the energy functional

$$
E_{\lambda}(u)=\frac{1}{2} \int_{\Omega}|\nabla u|^{2} d x+\frac{1}{\alpha+1} \int_{\Omega}|u|^{\alpha+1} d x-\frac{\lambda}{\beta+1} \int_{\Omega}|u|^{\beta+1} d x,
$$

where $H_{0}^{1}(\Omega)$ is the standard vanishing on the boundary Sobolev space. We are interested in ground states of $P(\alpha, \beta, \lambda)$ : i.e., a weak solution $u_{\lambda}$ of $P(\alpha, \beta, \lambda)$ which satisfies the inequality

$$
E_{\lambda}\left(u_{\lambda}\right) \leq E_{\lambda}\left(w_{\lambda}\right)
$$

\footnotetext{
*Corresponding Author: J.I. Díaz, Instituto de Matemática Interdisciplinar, Universidad Complutense de Madrid, 28040 Madrid, Spain, E-mail: jidiaz@ucm.es

J. Hernández, Instituto de Matemática Interdisciplinar, Universidad Complutense de Madrid, 28040 Madrid, Spain, E-mail: jesus.hernande@telefonica.net

Y.Sh. Ilyasov, Institute of Mathematics of UFRC RAS, Chernyshevsky str., 450008, Ufa, Rusia

Instituto de Matemática e Estatística, Universidade Federal de Goiás, 74001-970, Goiania, Brazil, E-mail: ilyasov02@gmail.com
} 
for any non-zero weak solution $w_{\lambda}$ of $P(\alpha, \beta, \lambda)$. Notice that in [1] the authors also use the term "ground state" with a different meaning.

Since the diffusion-reaction balance $-\Delta u=f(\lambda, u)$ involves the non-linear reaction term

$$
f(\lambda, u):=\lambda|u|^{\beta-1} u-|u|^{\alpha-1} u,
$$

and it is a non-Lipschitz function at zero (since $\alpha<1$ and $\beta<1$ ) important peculiar behavior of solutions of these problems arises. For instance, that may lead to the violation of the Hopf maximum principle on the boundary and the existence of compactly supported solutions as well as the so called flat solutions which correspond to weak solutions $u>0$ in $\Omega$ such that

$$
\frac{\partial u}{\partial v}=0 \text { on } \partial \Omega \text {, }
$$

where $v$ denotes the unit outward normal to $\partial \Omega$. When the additional information (1) holds but the weak solution may vanish in a positively measured subset of $\Omega$, i.e. $u \geq 0$ in $\Omega$, we shall call it as a compact support solution of $P(\alpha, \beta, \lambda)$ (sometimes also called as a free boundary solution, since the boundary of its support is not a priori known). Notice that in that case the support of $u$ is strictly included in $\bar{\Omega}$. If $u$ is a weak solution such that property (1) is not satisfied we shall call it as an "classical" weak solution (since, at least for the associated linear problem and for Lipschitz non-linear terms, the strong maximum principle due to Hopf, implies that (1) cannot be verified). However, we cannot exclude a priori the existence of solutions where (1) is only satisfied on part of $\partial \Omega$.

In what follows we shall use the following notation: any largest ball $B_{R(\Omega)}:=\left\{x \in \mathbb{R}^{N}:|x| \leq R(\Omega)\right\}$ contained in $\Omega$ will be denoted as an inscribed ball in $\Omega$. Our exact multiplicity results will concern the case of some classes of starshaped sets of $\mathbb{R}^{N}$ containing a finite number of different inscribed balls in $\Omega$.

For sufficiently large $\lambda$ the existence of a compactly supported solution of $P(\alpha, \beta, \lambda)$ follows from [2, 3] (see also for the case $N=1$, [4-9]. Indeed, by [2, 3, 8, 9] the equation in $P(\alpha, \beta, 1)$ considered in $\mathbb{R}^{N}$ has a unique (up to translation in $\mathbb{R}^{N}$ ) compactly supported solution $u^{\star}$, moreover $u^{\star}$ is radially symmetric such that $\operatorname{supp}\left(u^{\star}\right)=\bar{B}_{R^{*}}$ for some $R^{\star}>0$. Hence since the support of $u_{\sigma}^{\star}(x):=u^{\star}(x / \sigma), x \in B_{\sigma R^{*}}$ is contained in $\Omega$, for sufficiently small $\sigma$, the function $w_{\lambda}^{\sigma}(x)=\sigma^{-\frac{2}{1-\alpha}} \cdot u_{\sigma}^{*}(x)$ weakly satisfies $P(\alpha, \beta, \lambda)$ in $\Omega$ with $\lambda=\sigma^{-\frac{2(\beta-\alpha)}{1-\alpha}}$. However, it is not hard to show (see, e.g., Corollary 5.2 below) that, in general (for all sufficiently large $\lambda$ ), weak solutions $w_{\lambda}$ are not ground states.

On the other side, finding flat or compactly supported ground states is important in view of the study of non-stationary problems (see [10-13]).

The existence of flat and compact support ground states, for certain $\lambda^{\star}$ of $P(\alpha, \beta, \lambda)$ has been obtained in [14] (see also [11]). In the present paper we develop this result presenting here a sharper explanation of the main arguments of its proof. Furthermore, we shall offer here some more precise results on the behaviour of ground states depending on $\lambda$.

It is well known that the non-Lipschitz nonlinearities may entail the existence of a continuum of nonnegative compact supported solutions of elliptic boundary value problems. However the answer for the same question stated about ground states or "classical" weak solutions becomes unclear. Notice that this question is important in the investigation of stability solutions for non-stationary problems (see [10-12]). We recall that, as a matter of fact, flat solutions of $P\left(\alpha, \beta, \lambda^{\star}\right)$ only may arise if $\Omega$ is the ball $B_{R^{\star}}$ mentioned before. For the rest of domains, and values of $\lambda \geq \lambda^{\star}$, any weak solution which is not a "classical" weak solution should be radially symmetric and has compact support.

Let us state our main results. For given $u \in H_{0}^{1}(\Omega)$, the fibrering mappings are defined by $\phi_{u}(t)=E_{\lambda}(t u)$ so that from the variational formulation of $P(\alpha, \beta, \lambda)$ we know that $\left.\phi_{u}^{\prime}(t)\right|_{t=1}=0$ for solutions, where we use the notation

$$
\phi_{u}^{\prime}(t)=\frac{\partial}{\partial t} E_{\lambda}(t u)
$$

If we also define $\phi_{u}^{\prime \prime}(t)=\frac{\partial^{2}}{\partial t^{2}} E_{\lambda}(t u)$, then, since $\beta<1$ the equation $\phi_{u}^{\prime}(t)=0$ may have at most two nonzero roots $t_{\min }(u)>0$ and $t_{\max }(u)>0$ such that $\phi_{u}^{\prime \prime}\left(t_{\max }(u)\right) \leq 0, \phi_{u}^{\prime \prime}\left(t_{\min }(u)\right) \geq 0$ and $0<t_{\max }(u) \leq t_{\min }(u)$. This implies that any weak solution of $P(\alpha, \beta, \lambda)$ (any critical point of $E_{\lambda}(u)$ ) corresponds to one of the cases 
$t_{\min }(u)=1$ or $t_{\max }(u)=1$. However, it was discovered in [14] (see also [11, 12, 15]) that in case when we study flat or compactly supported solutions this correspondence essentially depends on the relation between $\alpha, \beta$ and $N$. Thus following this idea (from $[11,12,14,15]$, in the case $N \geq 3$, we consider the following subset of exponents

$$
\mathcal{E}_{s}(N):=\{(\alpha, \beta): 2(1+\alpha)(1+\beta)-N(1-\alpha)(1-\beta)<0,0<\alpha<\beta<1\} .
$$

The main property of $\varepsilon_{s}(N)$ is that for star-shaped domains $\Omega$ in $\mathbb{R}^{N}, N \geq 3$, if $(\alpha, \beta) \in \mathcal{E}_{s}(N)$, any ground state solution $u$ of $P(\alpha, \beta, \lambda)$ satisfies $\left.\phi_{u}^{\prime \prime}(t)\right|_{t=1}>0$ (see Lemma 2.2 below and [11, 14]).

Remark 1.1. In the cases $N=1,2$, one has $\mathcal{E}_{s}(N)=\emptyset$. Furthermore, this implies (see [11]) that if $N=1,2$ and $0<\alpha<\beta<1$, then any flat or compact support weak solution $u$ of $P(\alpha, \beta, \lambda)$ satisfies $\left.\phi_{u}^{\prime \prime}(t)\right|_{t=1}<0$.

In what follows we shall use the notations

$$
E_{\lambda}^{\prime}(u)=\left.\phi_{u}^{\prime}(t)\right|_{t=1}=\left.\frac{\partial}{\partial t} E_{\lambda}(t u)\right|_{t=1}, \quad E_{\lambda}^{\prime \prime}(u)=\left.\phi_{u}^{\prime \prime}(t)\right|_{t=1}=\left.\frac{\partial^{2}}{\partial t^{2}} E_{\lambda}(t u)\right|_{t=1}, \quad u \in H_{0}^{1}(\Omega) .
$$

Our first result is the following

Theorem 1.1. Let $N \geq 3$ and let $\Omega$ be a bounded strictly star-shaped domain in $\mathbb{R}^{N}$ with $C^{2}$-manifold boundary $\partial \Omega$. Assume that $(\alpha, \beta) \in \mathcal{E}_{s}(N)$. Then there exists $\lambda^{\star}>0$ such that for any $\lambda \geq \lambda^{\star}$ problem $P(\alpha, \beta, \lambda)$ possess a ground state $u_{\lambda}$. Moreover $E_{\lambda}^{\prime \prime}\left(u_{\lambda}\right)>0, u_{\lambda} \in C^{1, y}(\bar{\Omega})$ for some $y \in(0,1)$ and $u_{\lambda} \geq 0$ in $\Omega$. For any $\lambda<\lambda^{\star}$, problem $P(\alpha, \beta, \lambda)$ has no weak solution.

Our second main result deals with the existence (or not) of flat or compactly supported ground states.

Theorem 1.2. Under the same assumptions of the above theorem, there is a non-negative ground state $u_{\lambda^{\star}}$ which is flat or has compact support. Moreover, $u_{\lambda^{*}}$ is radially symmetric about some point of $\Omega$, and $\operatorname{supp}\left(u_{\lambda^{*}}\right)=\bar{B}_{R(\Omega)}$ is an inscribed ball in $\Omega$. For all $\lambda>\lambda^{\star}$, any ground state $u_{\lambda}$ of $P(\alpha, \beta, \lambda)$ is a "classical" weak solution.

Our last result deals with the multiplicity of solutions. Our main goal is to extend the results of [4] and [5] concerning the one-dimensional case. We also recall that the existence of what we call now "classical" weak solutions was proved in some previous papers in the literature. Existence of a smooth branch of such positive solutions was proved for $\lambda>\lambda^{*}$ in [16] by using a change of variables and then a continuation argument. The existence of at least two non-negative solutions in such a case was shown in [17] by using variational arguments and this result was improved in [18] showing that one of the solutions is actually positive, again by variational arguments. Most of these results are valid even in the singular case $-1<\alpha<\beta<1$.

In order to present our exact multiplicity results we introduce the geometrical reflection across a given hyperplane $H$ by the usual isometry $R_{H}: \mathbb{R}^{N} \rightarrow \mathbb{R}^{N}$. Remember that any point of $H$ is a fixed point of $R_{H}$. Now we shall introduce some classes of starshaped sets $\Omega$ for which we can obtain the exact multiplicity of flat stable ground solutions of problem $P\left(\alpha, \beta, \lambda^{\star}\right)$. We say that $\Omega$ is of Strictly Starshaped Class $m$, if it is a strictly starshaped domain and contains exactly $m$ inscribed balls of the same radius $R(\Omega)$ such that each of them can be obtained from any other by $k \in\{1, \ldots, m\}$ reflections of $\Omega$ across some hyperplanes $H_{i}, i=1, \ldots, k$.

Theorem 1.3. Assume $N \geq 3,(\alpha, \beta) \in \mathcal{E}_{s}(N)$. Let $\Omega$ be a domain of Strictly Starshaped Class $m>1$ with a $C^{2}$ manifold boundary $\partial \Omega$. Then there exist exactly $m$ stable nonnegative flat or compact supported ground states $u_{\lambda^{*}}^{1}, u_{\lambda^{*}}^{2}, \ldots, u_{\lambda^{*}}^{m}$ of problem $P\left(\alpha, \beta, \lambda^{\star}\right)$ and $m$ sets of “classical" ground states $\left(u_{\lambda_{n}}^{1}\right)_{n=1}^{\infty},\left(u_{\lambda_{n}}^{2}\right)_{n=1}^{\infty}, \ldots,\left(u_{\lambda_{n}}^{m}\right)_{n=1}^{\infty}$ of $P\left(\alpha, \beta, \lambda_{n}\right)$, , with $\lim _{n \rightarrow \infty} \lambda_{n}=\lambda^{\star}, \lambda_{n}>\lambda^{\star}, n=1,2, \ldots$ and such that $u_{\lambda_{n}}^{i} \rightarrow u_{\lambda^{*}}^{i}$, strongly in $H_{0}^{1}$ as $n \rightarrow \infty$, for any $i=1, \ldots, m$. 
The result concerning the exact number of stable non-negative flat (or compact support) solutions is, at the best of our knowledge, new, and the same can be said of the introduction of the classes of strictly star-shaped domains.

The second part of the statement of Theorem 1.3 seems to be a novelty as well. It provides a partial answer to the very general problem of knowing how a branch of positive solutions "leaving" at the interior of the positive cone in the space $C_{0}^{1}(\bar{\Omega})$ can "leave" this interior. It is known that in this case either $u(a)=0$ for some $a \in \Omega$ or $\frac{\partial u}{\partial v}(b)=0$ for some $b \in \partial \Omega$. For the problem we consider we know the answer in the onedimensional case ([4], [5]): if, say, $\Omega=(0,1)$, then $u(0)=u(1)=0$ and $u^{\prime}(0)=u^{\prime}(1)=0$ for such solutions $u(x)>0$ in $(0,1)$. For $N>1$ some partial results in the same direction are given in [10, 11, 14]. Here the situation is complicated due to the results by Kaper and Kwong $([8,9])$ showing that the supports of the compactly support solutions should be balls. We use here the symmetry properties of the domain in order to show the convergence of "classical" weak positive solutions to a flat (or compact support) solution supported on an inscribed ball of the domain.

Let us show how can be obtained some domains of Strictly Starshaped class $m$. We start by considering an initial bounded Lipschitz set $\Omega_{1}$ of $\mathbb{R}^{N}$ such that:

$$
\Omega_{1} \text { contains exactly one inscribed ball of radius } R\left(\Omega_{1}\right) \text {. }
$$

We also introduce the following notation: given a general open set $G$ of $\mathbb{R}^{N}$ we define $S[G]$ as the set of points $y \in G$ such that $G$ is strictly starshaped with respect to $y$. Then, the second condition we shall require to $\Omega_{1}$ is

$$
S\left[\Omega_{1}\right] \text { is not empty. }
$$

Then $\Omega$ belongs to the Strict Starshaped class 1 if there exists $\Omega_{1}$ satisfying (2) and (3) such that $\Omega=\Omega_{1}$. Now, let us show how we can obtain a domain of Strictly Starshaped class 2.

Let $\Omega_{1}$ be a domain of Strictly Starshaped class 1 and assume, additionally, that the set $S\left[\Omega_{1}\right]$ contains some other point different than $x_{1},\left\{x_{1}\right\} \varsubsetneqq S\left[\Omega_{1}\right]$, i.e.

$$
\text { there exists } y_{1} \in S\left[\Omega_{1}\right] \text { such that } y_{1} \neq x_{1} \text {. }
$$

Let now $\Omega_{2}:=R_{H\left(y_{1}\right)}\left(\Omega_{1}\right)$ be the reflected set of $\Omega_{1}$ across some hyperplane $H\left(y_{1}\right)$ containing the point $y_{1}$ such that

$$
\Omega_{1} \cup \Omega_{2} \text { contains exactly one inscribed ball of radius } R(\Omega) \text { of center } x_{2} \neq x_{1} \text {. }
$$

We now consider

$$
\Omega=\Omega_{1} \cup \Omega_{2} .
$$

Notice that, obviously, $\Omega$ is Strictly Starshaped class 1 with respect to $y_{1}$ (since $y_{1} \in S\left[\Omega_{1}\right]$ and any ray starting from $y_{1}$ is reflected to a ray linking $y_{1}$ with any other point of $\Omega_{2}$ ). Moreover, such a domain $\Omega$ verifies

$\Omega$ contains exactly two inscribed balls of radius $R(\Omega)$, with center at two different points $x_{i} \in \Omega, i=1,2$.

Thus $\Omega$ is a set of Strictly Starshaped class 2. Evidently we can repeat this construction with a domain of Strictly Starshaped class 2 and obtained a domain $\Omega$ of Strictly Starshaped class 3, etc.

We believe that we can iterate this process in a similar way until some number $m:=m(N) \geq 3$, which maybe depends on the dimension $N$. However we don't know how to prove this. Moreover we rise the following conjecture: For a given dimension $N$, there exists a number $m(N)$ such that for any $k=1,2, \ldots, m(N)$ there exists a domain of Strictly Starshaped class $k$ whereas there is no domain in $\mathbb{R}^{N}$ of Strictly Starshaped class $k$ with $k>m(N)$.

We emphasize that by Theorems 1.1, 1.2,1.3 we obtain the complete bifurcation diagram for the ground states of $P(\alpha, \beta, \lambda)$ for domains of Starshaped Class $m$. Indeed, the flat ground state $u_{\lambda^{\star}}$ corresponds to a fold 


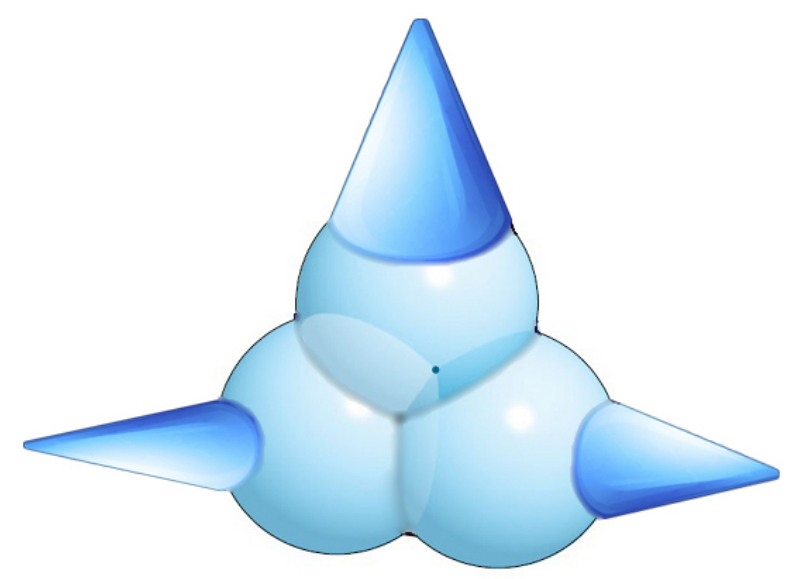

Fig. 1: Domain generating exactly three ground states

bifurcation point (or turning point) from which start $m+1$ different branches of weak solutions: on one hand, the branch of "classical" ground states $u_{\lambda}$, forming a branch of stable equilibria, and, on the other hand, $m$ branches formed by unstable compactly supported weak solutions, of the form $w_{\lambda}^{\sigma}\left(x: x_{0, j}\right)=\sigma^{-\frac{2}{1-\alpha}} \cdot u_{\lambda^{\star}}((x-$ $\left.x_{0, j}\right) / \sigma$ ) with $\lambda=\sigma^{-\frac{2(\beta-\alpha)}{1-\alpha}}$ (see Figure 1) and $m$ different points $x_{0, j}, j=1, \ldots, m$. Furthermore, we know a global information: the energy of $u_{\lambda^{*}}$ is the maximum among all the possible energies associated to any weak solution of $P(\alpha, \beta, \lambda)$ (due to the monotone dependence of $E_{\lambda}$ with respect to $\lambda$ ) and for $\lambda>\lambda^{\star}$ there are several ground states $u_{\lambda}=w_{\lambda}^{\sigma}$ with compact support and with an energy less than the one of $u_{\lambda^{*}}$.

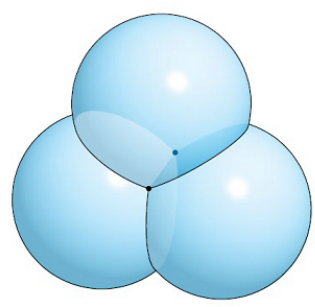

Fig. 2: Union of the supports of the three radially symmetric ground states corresponding to the domain given by Figure 1.

In the last part of the paper we consider the associate quasilinear parabolic problem of porous media type

$$
P P\left(m, \alpha, \beta, \lambda, v_{0}\right) \begin{cases}\left(|v|^{\frac{1}{m}-1} v\right)_{t}-\Delta v+|v|^{\alpha-1} v=\lambda|v|^{\beta-1} v & \text { in }(0,+\infty) \times \Omega \\ v=0 & \text { on }(0,+\infty) \times \partial \Omega \\ v(0, x)=v_{0}(x) & \text { on } \Omega,\end{cases}
$$

where $m>0$ and always under the structural assumption $0<\alpha<\beta<1$. The parabolic semilinear case $m=1$ was treated in the previous paper by the authors [11] and many other references were collected there. Notice that when $m \neq 1$ the problem usually appears in the literature formulated, equivalently, in terms of $w:=|v|^{\frac{1}{m}-1} v$

$$
\overline{P P}\left(m, a, b, \lambda, w_{0}\right) \quad \begin{cases}w_{t}-\Delta|w|^{m-1} w+|w|^{a-1} w=\lambda|w|^{b-1} w & \text { in }(0,+\infty) \times \Omega \\ w=0 & \text { on }(0,+\infty) \times \partial \Omega \\ w(0, x)=w_{0}(x) & \text { on } \Omega,\end{cases}
$$


with $a=\alpha m, b=\beta m$ and $w_{0}:=\left|v_{0}\right|^{\frac{1}{m}-1} v_{0}$. Notice that since the exponent $b$ may become greater than one, blow-up phenomena may occur depending on the initial datum and the balance between the exponents. Moreover, flat solutions over $\Omega$ can be extended by zero to the whole space $\mathbb{R}^{N}$ and so our treatment has important intersections with the study of the parabolic equation over $\mathbb{R}^{N}$. The pionering work in that direction was due, for the semilinear case $m=1$ and without the absorption term $|w|^{a-1} w$ to Fujita [19] who proved that for $b \in(0,1)$ all the solutions are globally defined in time, for $b \in(1,1+2 / N)$ all the solutions blow up in a finite time, whereas for $b \in(1+2 / N,+\infty)$ there exist both global solutions and blowing-up solutions according to the initial datum $w_{0}$. The quasilinear case $m>0$ was considered by many other authors, but most of them without the absorption term $|w|^{a-1} w$ (see, e.g., the monographs [20], [21]): in that case the Fujita exponent separating the three regimes is $m+2 / N$. The asymptotic behaviour for $t \rightarrow+\infty$ in presence of some absorption term, for a bounded domain $\Omega$, was also analyzed in the general sense of their associate attractors when the absorption term is linear $a=1$ ([22], [23], [24]).

Here we shall extend our previous results concerning the semilinear case and $\lambda=\lambda^{\star}$ by proving that the strong absorption term $|w|^{a-1} w$ modifies the above mentioned Fujita three regimes for exponent $b$ in the sense that, if $N \geq 3$, the stability region $\mathcal{E}_{s}(N)$ described before in terms of exponents $(\alpha, \beta)$ coincides with the equivalent region in terms of the exponents $(a, b)$ : so we shall prove that if $0<a<b<m$ and

$$
2(m+a)(m+b)-N(m-a)(m-b)<0,
$$

then the stationary flat solutions of $P P\left(m, \alpha, \beta, \lambda^{\star}, v_{0}\right)$ are stable (see Theorem 7.1).

We end this final section by applying some local energy methods, for the two cases $\lambda>\lambda^{\star}$ and $\lambda=\lambda^{\star}$, to give some information on the evolution and formation, respectively, of the free boundary given by the boundary of the support of the solution $v(t,$.$) when t$ increases. This provides a complementary information since by Theorem 1.1 (and the asymptotic behaviour results for $P P\left(m, \alpha, \beta, \lambda, v_{0}\right)$ ) we know that, as $t \rightarrow+\infty$, the support of $v(t,$.$) must converge to a ball of \mathbb{R}^{N}$, in the case $\lambda=\lambda^{\star}$, or to the whole domain $\bar{\Omega}$, if $\lambda>\lambda^{*}$, (the supports of one of the corresponding stationary solutions).

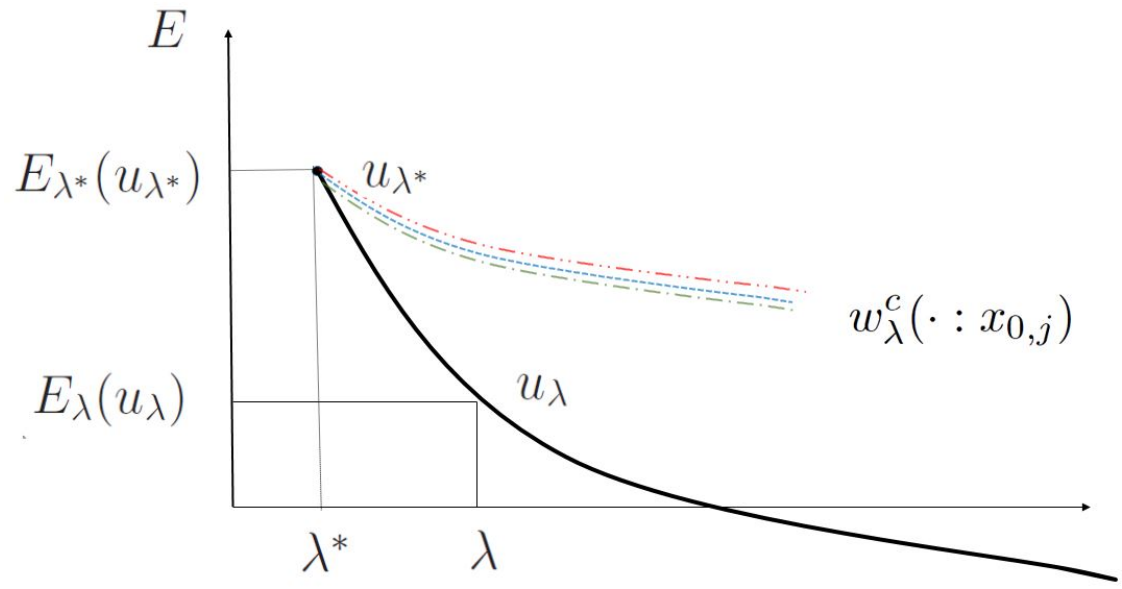

Fig. 3: Bifurcation diagram for the energy levels of ground states and compact support solutions. 


\section{Preliminaries}

In this section we give some preliminary results. In what follows $H_{0}^{1}:=H_{0}^{1}(\Omega)$ denotes the standard vanishing on the boundary Sobolev space. We can assume that its norm is given by

$$
\|u\|_{1}=\left(\int_{\Omega}|\nabla u|^{2} d x\right)^{1 / 2} .
$$

Denote

$$
P_{\lambda}(u):=\frac{1}{2^{\star}} \int_{\Omega}|\nabla u|^{2} \mathrm{~d} x+\frac{1}{\alpha+1} \int_{\Omega}|u|^{\alpha+1} \mathrm{~d} x-\lambda \frac{1}{\beta+1} \int_{\Omega}|u|^{\beta+1} \mathrm{~d} x,
$$

where

$$
2^{\star}=\frac{2 N}{N-2} \text { for } N \geq 3
$$

We will use the notation $P_{\lambda}^{\prime}(t u)=d P_{\lambda}(t u) / d t, t>0, u \in H_{0}^{1}$. From now on we suppose that the boundary $\partial \Omega$ is a $C^{2}$-manifold. As usual, we denote by $\mathrm{d} \sigma$ the surface measure on $\partial \Omega$. We need the Pohozhaev's identity for a weak solution of $P(\alpha, \beta, \lambda)$.

Lemma 2.1. Assume that $\partial \Omega$ is a $C^{2}$-manifold, $N \geq 3$. Let $u \in C^{1}(\bar{\Omega})$ be a weak solution of $P(\alpha, \beta, \lambda)$. Then there holds the Pohozaev identity

$$
P_{\lambda}(u)=-\frac{1}{2 N} \int_{\partial \Omega}\left|\frac{\partial u}{\partial v}\right|^{2}(x \cdot v(x)) \mathrm{d} \sigma(x) .
$$

For the proof see [11, 25] and [26, 27]. See also some related results in [26, 27].

Notice that

$$
E_{\lambda}(u)=P_{\lambda}(u)+\frac{1}{N} \int_{\Omega}|\nabla u|^{2} d x, \quad \forall u \in H_{0}^{1}(\Omega) .
$$

Assume $\Omega$ is strictly star-shaped with respect to a point $x_{0} \in \mathbb{R}^{N}$ (which will be identified as the origin of coordinates of $\mathbb{R}^{N}$ ). Observe that if $\Omega$ is a star-shaped (strictly star-shaped) domain with respect to the origin of $\mathbb{R}^{N}$, then $x \cdot v \geq 0(x \cdot v>0)$ for all $x \in \partial \Omega$. This and Lemma 2.1 imply

Corollary 2.1. Let $\Omega$ be a bounded star-shaped domain in $\mathbb{R}^{N}$ with a $C^{2}$-manifold boundary $\partial \Omega$. Then any weak solution $u \in C^{1}(\bar{\Omega})$ of $P(\alpha, \beta, \lambda)$ satisfies $P_{\lambda}(u) \leq 0$. Moreover, if $u$ is a flat solution or it has a compact support then $P_{\lambda}(u)=0$. Furthermore, in the case $\Omega$ is strictly star-shaped, the converse is also true: if $P_{\lambda}(u)=0$ and $u \in C^{1}(\bar{\Omega})$ is a weak solution of $P(\alpha, \beta, \lambda)$, then $u$ is flat or it has a compact support.

The proof of the following result can be found in [11, 14].

Lemma 2.2. Assume $N \geq 3$ and $(\alpha, \beta) \in \mathcal{E}_{s}(N)$.

(i) Let $u \in C^{1}(\bar{\Omega})$ be a flat or compact support weak solution of $P(\alpha, \beta, \lambda)$. Then $E_{\lambda}(u)>0$ and $E_{\lambda}^{\prime \prime}(u)>0$.

(ii) If $E_{\lambda}^{\prime}(u)=0, P_{\lambda}(u) \leq 0$ for some $u \in H_{0}^{1}(\Omega) \backslash 0$, then

$$
E_{\lambda}^{\prime \prime}(u)>0 .
$$

Remark 2.1. When $0<\beta<\alpha<1$, a case which is not considered in this paper, we have $E_{\lambda}^{\prime \prime}(u)>0$ and $P_{\lambda}(u)<0$ for any weak solution $u \in H_{0}^{1} \backslash 0$ of $P(\alpha, \beta, \lambda)$. In particular, in this case, any solution of $P(\alpha, \beta, \lambda)$ is a "classical" weak solution. The uniqueness of the solution was shown in [16].

In what follows we need also

Proposition 2.1. If $E_{\lambda}^{\prime}(t u)=0$ for $u \neq 0$, then $P_{\lambda}^{\prime}(t u)<0$. 
Proof Observe that,

$$
P_{\lambda}^{\prime}(t u)=\frac{N-2}{N} t \int_{\Omega}|\nabla u|^{2} \mathrm{~d} x-\lambda t^{\beta} \int_{\Omega}|u|^{\beta+1} \mathrm{~d} x+t^{\alpha} \int_{\Omega}|u|^{\alpha+1} \mathrm{~d} x=E_{\lambda}^{\prime}(t u)-\frac{2 t}{N} \int_{\Omega}|\nabla u|^{2} \mathrm{~d} x .
$$

Thus $E_{\lambda}^{\prime}(t u)=0$ entails $P_{\lambda}^{\prime}(t u)=-(2 t / N) \int|\nabla u|^{2} \mathrm{~d} x<0$.

\section{Auxiliary extremal values}

In this section we introduce some extremal values which will play an important role in the following. Some of these values, and the corresponding variational functionals, have been already introduced in [11, 14]. However, for our aims we shall introduce them using another approach which is more natural and easy.

Our approach will be based on using a nonlinear generalized Rayleigh quotient (see [28]). In fact, we can associate to problem $P(\alpha, \beta, \lambda)$ several nonlinear generalized Rayleigh quotients which may give useful information on the nature of the problem. In this paper we will deal with three of them.

First, let us consider the following Rayleigh's quotient [28]

$$
R^{0}(u)=\frac{\frac{1}{2} \int_{\Omega}|\nabla u|^{2} d x+\frac{1}{\alpha+1} \int_{\Omega}|u|^{\alpha+1} d x}{\frac{1}{\beta+1} \int_{\Omega}|u|^{\beta+1} d x}, u \neq 0 .
$$

Following [28], we consider

$$
r_{u}^{0}(t):=R^{0}(t u)=\frac{\frac{t^{1-\beta}}{2} \int_{\Omega}|\nabla u|^{2} d x+\frac{t^{\alpha-\beta}}{\alpha+1} \int_{\Omega}|u|^{\alpha+1} d x}{\frac{1}{\beta+1} \int_{\Omega}|u|^{\beta+1} d x}, t>0, \quad u \neq 0 .
$$

Notice that for any $u \neq 0$, and $\lambda \in \mathbb{R}$,

$$
\text { if }\left.R^{0}(u) \equiv r_{u}^{0}(t)\right|_{t=1}=\lambda \text {, then } E_{\lambda}(u)=0 .
$$

It is easy to see that $\partial r_{u}^{0}(t) / \partial t=0$ if and only if

$$
(1-\beta) \frac{t^{-\beta}}{2} \int_{\Omega}|\nabla u|^{2} d x+(\alpha-\beta) \frac{t^{\alpha-\beta-1}}{\alpha+1} \int_{\Omega}|u|^{\alpha+1} d x=0,
$$

and that the only solution to this equation is

$$
t_{0}(u)=\left(\frac{2(\beta-\alpha)}{(\alpha+1)(1-\beta)} \frac{\int_{\Omega}|u|^{\alpha+1} d x}{\int_{\Omega}|\nabla u|^{2} d x}\right)^{\frac{1}{1-\alpha}} .
$$

Let us emphasize that $t_{0}(u)$ is a value where the function $r_{u}^{0}(t)$ attains its global minimum. Substituting $t_{0}(u)$ into $r_{u}^{0}(t)$ we obtain the following nonlinear generalized Rayleigh quotient:

$$
\lambda_{0}(u)=\left.r_{u}^{0}\left(t_{0}(u)\right) \equiv R^{0}(t u)\right|_{t=t_{0}(u)}=c_{0}^{\alpha, \beta} \lambda(u),
$$

where

$$
c_{0}^{\alpha, \beta}=\frac{(1-\alpha)(\beta+1)}{(1-\beta)(1+\alpha)}\left(\frac{(1-\beta)(\alpha+1)}{2(\beta-\alpha)}\right)^{\frac{\beta-\alpha}{1-\alpha}},
$$

and

$$
\lambda(u)=\frac{\left(\int_{\Omega}|u|^{\alpha+1} d x\right)^{\frac{1-\beta}{1-\alpha}}\left(\int_{\Omega}|\nabla u|^{2} d x\right)^{\frac{\beta-\alpha}{1-\alpha}}}{\int_{\Omega}|u|^{\beta+1} d x} .
$$

See Figure 4.

It is not hard to prove (see, e.g., page 400 of [29]) that 


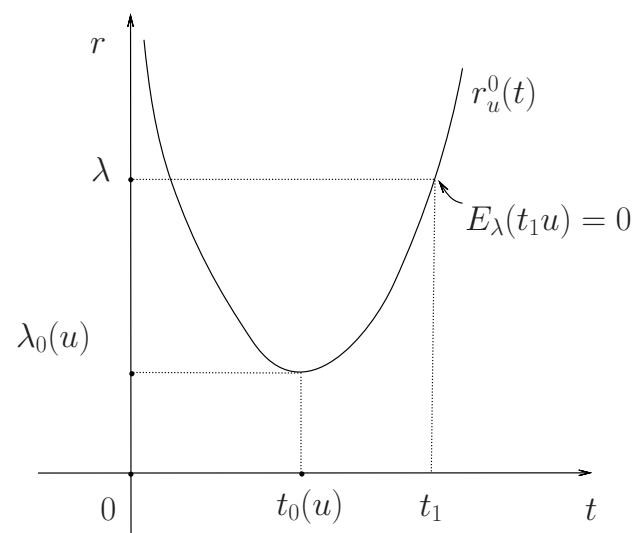

Fig. 4

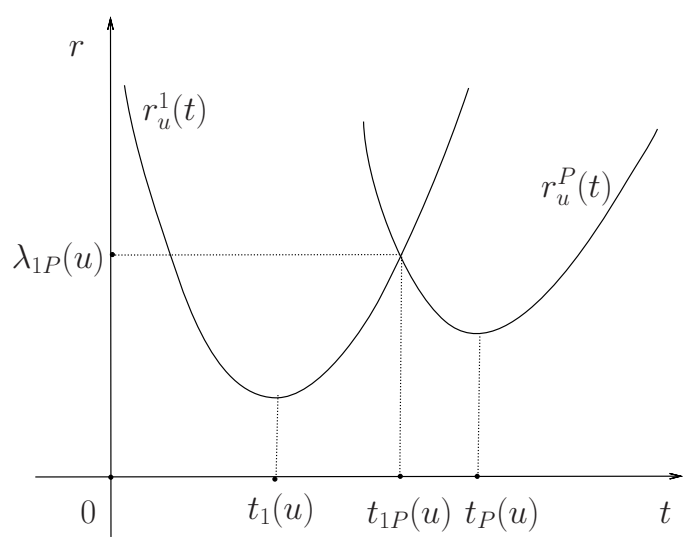

Fig. 5

Proposition 3.1. The map $\lambda(\cdot): H_{0}^{1}(\Omega) \backslash 0 \rightarrow \mathbb{R}$ is a $C^{1}$-functional.

Consider the following extremal value of $\lambda_{0}(u)$

$$
\Lambda_{0}=\inf _{u \in H_{0}^{1}(\Omega) \backslash 0} \lambda_{0}(u) .
$$

Using Sobolev's and Hölder's inequalities (see, e.g., [14]) it can be shown that

$$
0<\Lambda_{0}<+\infty .
$$

By the above construction and using (9) it is not hard to prove the following

Proposition 3.2. (i) If $\lambda<\Lambda_{0}$, then $E_{\lambda}(u)>0$ for any $u \neq 0$,

(ii) For any $\lambda>\Lambda_{0}$ there is $u \in H_{0}^{1}(\Omega) \backslash 0$ such that $E_{\lambda}(u)<0, E_{\lambda}^{\prime}(u)=0$.

In what follows we shall use the following result:

Proposition 3.3. Let $u$ be a critical point of $\lambda_{0}(u)$ at some critical value $\bar{\lambda}$, i.e. $D_{u} \lambda_{0}(u)=0, \bar{\lambda}=\lambda_{0}(u)$. Then $D_{u} E_{\bar{\lambda}}(u)=0$ and $E_{\bar{\lambda}}(u)=0$.

Proof Observe that

$$
D_{u} \lambda_{0}(u)(\phi)=D_{u} r_{u}^{0}\left(t_{0}(u)\right)(\phi)+\frac{\partial}{\partial t} r_{u}^{0}\left(t_{0}(u)\right)\left(D_{u} t_{0}(u)(\phi)\right)=0, \quad \forall \phi \in C_{0}^{\infty}(\Omega) .
$$

Hence, since $\partial r_{u}^{0}(t) /\left.\partial t\right|_{t=t_{0}(u)}=0$, we get

$$
D_{u} r_{u}^{0}\left(t_{0}(u)\right)(\phi)=\left.t_{0}(u) \cdot D_{w} R^{0}(w)\right|_{w=t_{0}(u) u}(\phi)=0, \quad \forall \phi \in C_{0}^{\infty}(\Omega) .
$$

Now taking into account that the equality $\bar{\lambda}=\lambda_{0}(u)$ implies $E_{\bar{\lambda}}(u)=0$, we obtain

$$
0=\left.D_{w} R^{0}(w)\right|_{w=t_{0}(u) u}=\left.\frac{1}{\int_{\Omega}|w|^{\beta+1} d x} \cdot D_{w} E_{\bar{\lambda}}(w)\right|_{w=t_{0}(u) u},
$$

which yields the proof.

We shall need also the following Rayleigh's quotients:

$$
\begin{aligned}
& R^{P}(u)=\frac{\frac{1}{2^{\star}} \int_{\Omega}|\nabla u|^{2} \mathrm{~d} x+\frac{1}{\alpha+1} \int_{\Omega}|u|^{\alpha+1} d x}{\frac{1}{\beta+1} \int_{\Omega}|u|^{\beta+1} d x}, \\
& R^{1}(u)=\frac{\int_{\Omega}|\nabla u|^{2} \mathrm{~d} x+\int_{\Omega}|u|^{\alpha+1} d x}{\int_{\Omega}|u|^{\beta+1} d x}, u \neq 0 .
\end{aligned}
$$


Notice that for any $u \neq 0$ and $\lambda \in \mathbb{R}$,

$$
R^{P}(u)=\lambda \Leftrightarrow P_{\lambda}(u)=0 \text { and } R^{1}(u)=\lambda \Leftrightarrow E_{\lambda}^{\prime}(u)=0 .
$$

Let $u \neq 0$. Consider $r_{u}^{P}(t):=R^{P}(t u), r_{u}^{1}(t):=R^{1}(t u), t>0$. Then, arguing as above for $r_{u}^{0}(t)$, it can be shown that each of these functions attains its global minimum at some point, $t_{P}(u)$ and $t_{1}(u)$, respectively. Moreover, it is easily seen that the following equation

$$
r_{u}^{P}(t)=r_{u}^{1}(t), \quad t>0,
$$

has a unique solution

$$
t_{1 P}(u)=\left(\frac{2^{\star}(\beta-\alpha)}{\left(2^{\star}-\beta-1\right)(\alpha+1)} \frac{\int_{\Omega}|u|^{\alpha+1} d x}{\int_{\Omega}|\nabla u|^{2} d x}\right)^{\frac{1}{1-\alpha}} .
$$

Thus, we have the next nonlinear generalized Rayleigh quotient

$$
\lambda_{1 P}(u):=r_{u}^{P}\left(t_{1 P}(u)\right)=r_{u}^{1}\left(t_{1 P}(u)\right) .
$$

It is easily to seen that $\lambda_{1 P}(u)=c_{1 P}^{\alpha, \beta} \lambda(u)$, where

$$
c_{1 P}^{\alpha, \beta}=\frac{(\beta+1)\left(2^{\star}-\alpha+1\right)}{(\beta-\alpha) 2^{\star}}\left(\frac{2^{\star}(\beta-\alpha)}{\left(2^{\star}-\beta-1\right)(\alpha+1)}\right)^{\frac{\beta-\alpha}{1-\alpha}} .
$$

Notice that

$$
P_{\lambda_{1 P}(u)}\left(t_{1 P}(u) u\right)=0, \quad E_{\lambda_{1 P}(u)}^{\prime}\left(t_{1 P}(u) u\right)=0, \quad \forall u \neq 0 .
$$

Consider

$$
\Lambda_{1 P}=\inf _{u \neq 0} \lambda_{1 P}(u) .
$$

Using Sobolev's and Hölder's inequalities it can be shown (see, e.g., [14]) that

$$
0<\Lambda_{1 P}<+\infty .
$$

Moreover we have (see Figure 5):

Proposition 3.4. For any $u \neq 0$,

(i) $r_{u}^{P}(t)>r_{u}^{1}(t)$ if $t \in\left(0, t_{1 P}(u)\right)$ and $r_{u}^{P}(t)<r_{u}^{1}(t)$ if $t \in\left(t_{1 P}(u),+\infty\right)$;

(ii) $t_{1}(u)<t_{1 P}(u)<t_{P}(u)$.

Proof Observe that $r_{u}^{P}(t) / r_{u}^{1}(t) \rightarrow \frac{\beta+1}{\alpha+1}>1$ as $t \rightarrow 0$. Hence, from the uniqueness of $t_{1 P}(u)$ we obtain (i).

By (17) we have $E_{\lambda_{1 P}(u)}^{\prime}\left(t_{1 P}(u) u\right)=0$. Therefore Proposition 2.1 implies $\frac{d}{d t} P_{\lambda_{1 P}(u)}\left(t_{1 P}(u) u\right)<0$. Hence and since

$$
\left.\frac{d}{d t} r_{u}^{P}(t)\right|_{t=t_{1 P}(u)}=\left.\frac{\beta+1}{\int|t u|^{\beta+1} d x} \cdot \frac{d}{d t} P_{\lambda_{1 P}(u)}(t u)\right|_{t=t_{1 P}(u)},
$$

we conclude that $\left.\frac{d}{d t} r_{u}^{P}(t)\right|_{t=t_{1 P}(u)}<0$. Now taking into account that $t_{P}(u)$ is a point of global minimum of $r_{u}^{P}(t)$ we obtain that $t_{1 P}(u)<t_{P}(u)$. To prove of $t_{1}(u)<t_{1 P}(u)$, first observe that

$$
\left.\frac{d}{d t} r_{u}^{1}(t)\right|_{t=t_{1 P}(u)}=\left.\frac{1}{\int_{\Omega}|t u|^{\beta+1} d x} \cdot E_{\lambda_{1 P}(u)}^{\prime \prime}(t u)\right|_{t=t_{1 P}(u)},
$$

and that by Lemma 2.2 the equalities $E_{\lambda_{1 P}(u)}^{\prime}\left(t_{1 P}(u) u\right)=0, P_{\lambda_{1 P}(u)}\left(t_{1 P}(u) u\right)=0$ imply $E_{\lambda_{1 P}(u)}^{\prime \prime}\left(t_{1 P}(u) u\right)>0$. Thus $\frac{d}{d t} r_{u}^{1}\left(t_{1 P}(u)\right)>0$ and the proof follows.

Corollary 3.1. (i) If $\lambda<\Lambda_{1 P}$ and $E_{\lambda}^{\prime}(u)=0$, then $P_{\lambda}(u)>0$.

(ii) For any $\lambda>\Lambda_{1 P}$, there exists $u \in H_{0}^{1} \backslash 0$ such that $E_{\lambda}^{\prime}(u)=0$ and $P_{\lambda}(u) \leq 0$. 
Proof (i). Let $u \in H_{0}^{1} \backslash 0$. Assume $\lambda<\lambda_{1 P}(u)$ such that $E_{\lambda}^{\prime}(u)=0$. Then in view of (17) we have $R^{1}(u)=$ $\lambda<\lambda_{1 P}(u)$. If $\Omega$ is starshaped we know that $P_{\lambda}(u) \leq 0$ and then $E_{\lambda}^{\prime}(u) \neq 0$. Thus (ii), Proposition 3.4 yields $1 \equiv t_{1}(u)<t_{1 P}(u)$ and therefore by (i), Proposition 3.4 we have $r_{u}^{P}(1)>r_{u}^{1}(1)=\lambda$. Thus by $(7)$ we get $P_{\lambda}(u)>0$. The proof of (ii) is similar to (i).

Corollary 3.2. $\Lambda_{1 P}<\Lambda_{0}$.

Proof Suppose that $\Lambda_{0}<\Lambda_{1 P}$. From Proposition 3.2 for any $\lambda \in\left(\Lambda_{0}, \Lambda_{1 P}\right)$, there exists $u \neq 0$ such that $E_{\lambda}(u)<0$ and $E_{\lambda}^{\prime}(u)=0$. By Corollary 3.1, the equality $E_{\lambda}^{\prime}(u)=0$ entails $P_{\lambda}(u)>0$. Hence by (6) we have $E_{\lambda}(u)>P_{\lambda}(u)>0$, i.e., we get a contradiction. The equality $\Lambda_{0}=\Lambda_{1 P}$ is impossible since $c_{1 P}^{\alpha, \beta} \neq c_{0}^{\alpha, \beta}$.

Corollary 3.3. Let $\Omega$ be a bounded star-shaped domain in $\mathbb{R}^{N}$ with $C^{2}$-manifold boundary $\partial \Omega$. Then for any $\lambda<\Lambda_{1 P}$ equation $P(\alpha, \beta, \lambda)$ cannot have weak solutions.

Proof Let $\lambda<\Lambda_{1 P}$. Assume conversely that there exists a weak solution $u$. By the regularity of solutions of elliptic equations, $u \in C^{1}(\bar{\Omega})$. Then since $E_{\lambda}^{\prime}(u)=0$, by Corollary 3.1 we have $P_{\lambda}(u)>0$. However by Corollary 2.1, any weak solution $u \in C^{1}(\bar{\Omega})$ of $P(\alpha, \beta, \lambda)$ satisfies $P_{\lambda}(u) \leq 0$. Thus we get a contradiction.

\section{Main constrained minimization problem}

Consider the constrained minimization problem:

$$
\hat{E}_{\lambda}:=\min _{u \in M_{\lambda}} E_{\lambda}(u)
$$

where

$$
M_{\lambda}:=\left\{u \in H_{0}^{1} \backslash 0: E_{\lambda}^{\prime}(u)=0, P_{\lambda}(u) \leq 0\right\} .
$$

Observe that any weak solution of $P(\alpha, \beta, \lambda)$ belongs to $M_{\lambda}$, such as it follows from Corollary 2.1. Hence if $\hat{E}_{\lambda}=E_{\lambda}\left(u_{\lambda}\right)$, in (24), for some solution $u_{\lambda}$ of $P(\alpha, \beta, \lambda)$, then $u_{\lambda}$ is a ground state.

Proposition 4.1. $M_{\lambda} \neq \emptyset$ for any $\lambda>\Lambda_{1 P}$.

Proof Let $\lambda>\Lambda_{1 P}$. Consider the function $\lambda_{1 P}(\cdot): H_{0}^{1} \backslash 0 \rightarrow \mathbb{R}$. By Proposition 3.1 this is a continuous functional. Hence there is $u \in H_{0}^{1} \backslash 0$ such that $\Lambda_{1 P}<\lambda_{1 P}(u)<\lambda$. Since by (21) we have $P_{\lambda_{1 P}(u)}\left(t_{1 P}(u) u\right)=0$, $E_{\lambda_{1 P}(u)}^{\prime}\left(t_{1 P}(u) u\right)=0$, it follows $P_{\lambda}\left(t_{1 P}(u) u\right)<0, E_{\lambda}^{\prime}\left(t_{1 P}(u) u\right)<0$. Hence there is $t_{\min }(u)>t_{1 P}(u)$ such that $E_{\lambda}^{\prime}\left(t_{\min }(u) u\right)=0$. In view that $P_{\lambda}^{\prime}(t u)=E_{\lambda}^{\prime}(t u)-(2 t / N) \int|\nabla u|^{2}$ for any $t>0$ we have $P_{\lambda}^{\prime}\left(t_{\min }(u) u\right)<0$ which implies that $P_{\lambda}\left(t_{\min }(u) u\right)<0$. Thus $t_{\min }(u) u \in M_{\lambda}$.

Lemma 4.1. For any $\lambda>\Lambda_{1 P}$ there exists a minimizer $u_{\lambda}$ of problem (24), i.e., $E_{\lambda}\left(u_{\lambda}\right)=\hat{E}_{\lambda}$ and $u_{\lambda} \in M_{\lambda}$.

Proof Let $\lambda>\Lambda_{1 P}$. Then $M_{\lambda}$ is bounded. Indeed, if $u \in M_{\lambda}$, then

$$
\frac{1}{2^{\star}} \int_{\Omega}|\nabla u|^{2} \mathrm{~d} x+\frac{1}{\alpha+1} \int_{\Omega}|u|^{\alpha+1} \mathrm{~d} x \leq \lambda \frac{1}{\beta+1} \int_{\Omega}|u|^{\beta+1} \mathrm{~d} x \leq c \lambda \frac{1}{\beta+1}\|u\|_{1}^{\beta+1} .
$$

From here $\|u\|_{1} \leq C<+\infty, \forall u \in M_{\lambda}$. Now, if $\left(u_{m}\right)$ is a minimizing sequence of (24), then it is bounded and there exists a subsequence, denoted again $\left(u_{m}\right)$, which converges $u_{m} \rightarrow u_{0}$ weakly in $H_{0}^{1}$ and strongly $u_{m} \rightarrow u_{0}$ in 
$L^{q}, 1<q<2^{\star}$. We claim that $u_{m} \rightarrow u_{0}$ strongly in $H_{0}^{1}$. If not, $\left\|u_{0}\right\|_{1}<\liminf _{m \rightarrow \infty}\left\|u_{m}\right\|_{1}$ and this implies

$$
\begin{aligned}
\int_{\Omega}\left|\nabla u_{0}\right|^{2} \mathrm{~d} x+ & \int_{\Omega}\left|u_{0}\right|^{\alpha+1} \mathrm{~d} x-\lambda \int_{\Omega}\left|u_{0}\right|^{\beta+1} \mathrm{~d} x< \\
& \liminf _{m \rightarrow \infty}\left(\int_{\Omega}\left|\nabla u_{m}\right|^{2} \mathrm{~d} x+\int_{\Omega}\left|u_{m}\right|^{\alpha+1} \mathrm{~d} x-\lambda \int_{\Omega}\left|u_{m}\right|^{\beta+1} \mathrm{~d} x\right)=0
\end{aligned}
$$

since $E_{\lambda}^{\prime}\left(u_{m}\right)=0, m=1,2, \ldots$. Hence $u_{0} \neq 0$ and $E_{\lambda}^{\prime}\left(u_{0}\right)<0$. Then there exists $y>1$ such that $E_{\lambda}^{\prime}\left(y u_{0}\right)=0$ and $E_{\lambda}\left(y u_{0}\right)<E_{\lambda}\left(u_{0}\right)<\hat{E}_{\lambda}$. By Proposition 2.1, $E_{\lambda}^{\prime}\left(y u_{0}\right)=0$ implies $P_{\lambda}^{\prime}\left(y u_{0}\right)<0$. From this and since

$$
P_{\lambda}\left(u_{0}\right)<\liminf _{m \rightarrow \infty} P_{\lambda}\left(u_{m}\right) \leq 0,
$$

we conclude that $P_{\lambda}\left(y u_{0}\right)<0$. Thus $y u_{0} \in M_{\lambda}$ and $E_{\lambda}\left(y u_{0}\right)<\hat{E}_{\lambda}$, which is a contradiction.

\subsection{Existence of a flat or compact support ground state $\boldsymbol{u}_{\lambda^{*}}$}

Let $\lambda>\Lambda_{1 P}$, then by Lemma 4.1 there exists a minimizer $u_{\lambda}$ of (24). Notice $\operatorname{since} \min \{\alpha, \beta\}>0, E_{\lambda}(u)$ and $E_{\lambda}^{\prime}(u), P_{\lambda}(u)$ are $C^{1}$-functionals on $H_{0}^{1}(\Omega)$. Hence we may apply Lagrange multipliers rule (see, e.g., page 417 of [29]) and thereby there exist Lagrange multipliers $\mu_{0}, \mu_{1} \mu_{2}$ such that $\left|\mu_{0}\right|+\left|\mu_{1}\right|+\left|\mu_{2}\right| \neq 0, \mu_{2} \geq 0$ (since the unilateral constraint) and

$$
\begin{aligned}
& \mu_{0} D_{u} E_{\lambda}\left(u_{\lambda}\right)+\mu_{1} D_{u} E_{\lambda}^{\prime}\left(u_{\lambda}\right)+\mu_{2} D_{u} P_{\lambda}\left(u_{\lambda}\right)=0, \\
& \mu_{2} P_{\lambda}\left(u_{\lambda}\right)=0 .
\end{aligned}
$$

Proposition 4.2. Assume $(\alpha, \beta) \in \mathcal{E}_{s}(N)$. Let $\lambda>\Lambda_{1 P}$ and $u_{\lambda} \in H_{0}^{1}$ be a minimizer in (24) such that $P_{\lambda}\left(u_{\lambda}\right)<0$. Then $u_{\lambda}$ is a weak solution to $P(\alpha, \beta, \lambda)$.

Proof Since $P_{\lambda}\left(u_{\lambda}\right)<0$, equality (26) implies $\mu_{2}=0$. Moreover, since $(\alpha, \beta) \in \mathcal{E}_{s}(N)$, (ii), Lemma 2.2 implies that $E_{\lambda}^{\prime \prime}\left(u_{\lambda}\right)>0$. Testing $(25)$ by $u_{\lambda}$ we get $0=\mu_{0} E_{\lambda}^{\prime}\left(u_{\lambda}\right)=\mu_{1} E_{\lambda}^{\prime \prime}\left(u_{\lambda}\right)$. But $E_{\lambda}^{\prime \prime}\left(u_{\lambda}\right) \neq 0$ and therefore $\mu_{1}=0$. Thus, $D_{u} E_{\lambda}\left(u_{\lambda}\right)=0$, that is $u_{\lambda}$ weakly satisfies $P(\alpha, \beta, \lambda)$. This completes the proof. Introduce

$$
Z:=\left\{\lambda \in\left(\Lambda_{1 P},+\infty\right): P_{\lambda}\left(u_{\lambda}\right)<0, u_{\lambda} \in M_{\lambda} \text { s.t. } E_{\lambda}\left(u_{\lambda}\right)=\hat{E}_{\lambda}\right\} .
$$

Proposition 4.3. $Z$ is a non-empty open subset of $\left(\Lambda_{1 P},+\infty\right)$.

Proof Notice that by Lemma 4.1, for any $\lambda>\Lambda_{1 P}$ there exists $u_{\lambda} \in M_{\lambda}$ such that $E_{\lambda}\left(u_{\lambda}\right)=\hat{E}_{\lambda}$. To prove that $Z \neq \emptyset$, we show that $\left[\Lambda_{0},+\infty\right) \subset Z$. Take $\lambda \geq \Lambda_{0}$. Then in view of (ii), Proposition 3.2 we have $\hat{E}_{\lambda} \leq 0$. Thus $E_{\lambda}\left(u_{\lambda}\right) \leq 0$, for any $u_{\lambda} \in M_{\lambda}$ such that $E_{\lambda}\left(u_{\lambda}\right)=\hat{E}_{\lambda}$. In view of (6) we have $E_{\lambda}\left(u_{\lambda}\right)>P_{\lambda}\left(u_{\lambda}\right)$ and therefore $P_{\lambda}\left(u_{\lambda}\right)<0, \forall u_{\lambda} \in M_{\lambda}$ such that $E_{\lambda}\left(u_{\lambda}\right)=\hat{E}_{\lambda}$. Thus $\lambda \in Z$.

Let us show that $Z$ is an open subset of $\left(\Lambda_{1 P},+\infty\right)$. Notice that if $Z=\left(\Lambda_{1 P},+\infty\right)$, then $Z$ is an open subset of $\left(\Lambda_{1 P},+\infty\right)$ by the definition.

Assume $Z \neq\left(\Lambda_{1 P},+\infty\right)$. Let $\lambda \in Z$. Suppose, contrary to our claim, that there is a sequence $\left(\lambda_{m}\right) \subset\left(\Lambda_{1 P},+\infty\right) \backslash Z$ such that $\lambda_{m} \rightarrow \lambda$ as $m \rightarrow \infty$. Then there is a sequence of solutions $\left(u_{\lambda_{m}}\right)$ of (24) such that $P_{\lambda_{m}}\left(u_{\lambda_{m}}\right)=0$. Then by Lemma A.1 (see Appendix I), there exists a minimizer $u_{\lambda}$ of (24) and a subsequence, still denoted by $\left(u_{\lambda_{m}}\right)$, such that $u_{\lambda_{m}} \rightarrow u_{\lambda}$ strongly in $H_{0}^{1}$ as $m \rightarrow+\infty$. However, then $P_{\lambda}\left(u_{\lambda}\right)=0$, which contradicts the assumption $\lambda \in Z$.

Set

$$
\lambda^{\star}:=\inf Z
$$

Lemma 4.2. There exists a minimizer $u_{\lambda^{*}}$ of (24) which is a flat or a compact support non-negative ground state of $P\left(\alpha, \beta, \lambda^{*}\right)$. Furthermore, $\Lambda_{1 P}<\lambda^{*}$ and there exists a set of "classical" non-negative ground states $\left(u_{\lambda_{n}}\right)_{n=1}^{\infty}$ of $P\left(\alpha, \beta, \lambda_{n}\right)$, with $\lambda_{n} \downarrow \lambda^{\star}$ as $n \rightarrow \infty$, such that $u_{\lambda_{n}} \rightarrow u_{\lambda^{\star}}$ strongly in $H_{0}^{1}$ as $n \rightarrow \infty$. 
Proof Since $Z$ is an open set, we can find a sequence $\lambda_{n} \in Z, n=1,2, \ldots$ such that $\lambda_{n} \rightarrow \lambda^{\star}$ as $n \rightarrow \infty$. By the definition of $Z$ for any $n=1,2, \ldots$ we can find a minimizer $u_{\lambda_{n}}$ of (24) such that $P_{\lambda_{n}}\left(u_{\lambda_{n}}\right)<0$. Then Proposition 4.2 yields that $u_{\lambda_{n}}$ weakly satisfies $P\left(\alpha, \beta, \lambda_{n}\right), n=1,2, \ldots$. Moreover by Corollary 2.1, $u_{\lambda_{n}}$ is a "classical" weak solution of $P\left(\alpha, \beta, \lambda_{n}\right), n=1,2, \ldots$. Since $E_{\lambda}(|u|)=E_{\lambda}(u), E_{\lambda}^{\prime}(|u|)=E_{\lambda}^{\prime}(u)=0, P_{\lambda}(|u|)=P_{\lambda}(u)$ for any $u \in H_{0}^{1}$ we may assume that $u_{\lambda_{n}} \geq 0, n=1,2, \ldots$. Furthermore, since $\hat{E}_{\lambda_{n}}=E_{\lambda_{n}}\left(u_{\lambda_{n}}\right), u_{\lambda_{n}}$ is a ground state of $P\left(\alpha, \beta, \lambda_{n}\right), n=1,2, \ldots$. Thus we have a set of "classical" non-negative ground states $\left(u_{\lambda_{n}}\right)_{n=1}^{\infty}$ of $P\left(\alpha, \beta, \lambda_{n}\right)$, $n=1,2, \ldots$

By Lemma A.1 (see Appendix I), there exists a minimizer $u_{\lambda^{*}}$ of (24) and the subsequence, still denoted by $\left(u_{\lambda_{n}}\right)$, such that $u_{\lambda_{n}} \rightarrow u_{\lambda^{\star}}$ strongly in $H_{0}^{1}$ as $\lambda_{n} \rightarrow \lambda^{\star}$. This implies that $u_{\lambda^{\star}}$ is a non-negative solution of $P\left(\alpha, \beta, \lambda^{\star}\right)$ and $P_{\lambda^{\star}}\left(u_{\lambda^{\star}}\right) \leq 0$. Furthermore, since $u_{\lambda^{\star}}$ is a minimizer of (24), it is a ground state of $P\left(\alpha, \beta, \lambda^{\star}\right)$.

Let us show that $\Lambda_{1 P}<\lambda^{\star}$. To obtain a contradiction suppose that $\Lambda_{1 P}=\lambda^{\star}$. Then $\Lambda_{1 P}=\lambda_{1 P}\left(u_{\lambda^{\star}}\right)$ and $u_{\lambda^{\star}}$ is a minimizer of (22). Since $\lambda_{1 P}(u)=c^{\alpha, \beta} \lambda_{0}(u)$, where $c^{\alpha, \beta}=c_{1 P}^{\alpha, \beta} / c_{0}^{\alpha, \beta}, u_{\lambda^{\star}}$ is also a critical point of $\lambda_{0}(u)$ with value $\Lambda_{0}$. Then by Proposition 3.3, $u_{\lambda^{\star}}$ satisfies $P\left(\alpha, \beta, \Lambda_{0}\right)$. However, by the construction $u_{\lambda^{\star}}$ satisfies $P\left(\alpha, \beta, \lambda^{\star}\right)$. Notice that by Corollary 3.2, $\Lambda_{0}>\Lambda_{1 P}=\lambda^{\star}$. Thus we get a contradiction.

Observe that $P_{\lambda^{*}}\left(u_{\lambda^{*}}\right)=0$. Indeed, if $P_{\lambda^{*}}\left(u_{\lambda^{*}}\right)<0$, then $\lambda^{\star} \in Z$. But this is impossible since $Z$ is an open subset of $\left(\Lambda_{1 P},+\infty\right)$.

A global (up to the boundary) regularity result (see [30]) yields that $u_{\lambda} \in C^{1, \beta}(\bar{\Omega}), \lambda \in\left[\lambda^{*},+\infty\right.$ ) for some $\beta \in(0,1)$. Thus we may apply Corollary 2.1 which yields that $u_{\lambda^{\star}}$ is flat or compactly supported in $\Omega$.

\section{On the radially symmetric property}

We need the following result that has been proved in $[3,8,9]$.

Lemma 5.1. Assume $0<\alpha<\beta<1$. Let $u$ be a non-negative $C^{1}$ distribution solution of

$$
-\Delta u+u^{\alpha}=u^{\beta} \text { in } \mathbb{R}^{N}
$$

with connected support. Then the support of $u$ is a ball and $u$ is radially symmetric about the center.

Furthermore, equation $\mathrm{E} q(\alpha, \beta, 1)$ admits at most one radially symmetric compact support solution.

We denote by $R^{\star}$ the radius of the supporting ball $B_{R^{\star}}$ of the unique (up to translation in $\mathbb{R}^{N}$ ) compact support solution of $E q(\alpha, \beta, 1)$, i.e., it is the unique flat solution of $P(\alpha, \beta, 1)$ for $\Omega=B_{R^{*}}$.

It is easy to see, from Lemma 5.1, that the function $u_{\lambda}^{\star}(x):=\sigma^{-\frac{2}{1-\alpha}} \cdot u^{\star}(x / \sigma)$ is the unique flat solution of $P(\alpha, \beta, \lambda)$ with $\lambda=\sigma^{-\frac{2(\beta-\alpha)}{1-\alpha}}$ and $\Omega=B_{\sigma R^{*}}$.

Proposition 5.1. Assume $u_{\lambda} \in C^{1}(\bar{\Omega})$ is a non-negative ground state of $P(\alpha, \beta, \lambda)$ which has compact support in $\Omega$. Then $u_{\lambda}$ is radially symmetric about some origin $0 \in \Omega$, and $\operatorname{supp}\left(u_{\lambda}\right)=\overline{B_{R(\Omega)}}$ is a inscribed ball in $\bar{\Omega}$.

Proof Observe that any compact support function $u_{\lambda}$ from $C^{1}(\bar{\Omega})$ can be extended to $\mathbb{R}^{N}$ as

$$
\begin{cases}\tilde{u}_{\lambda}=u_{\lambda} & \text { in } \Omega \\ \tilde{u}_{\lambda}=0 & \text { in } \mathbb{R}^{N} \backslash \Omega .\end{cases}
$$

Then $\tilde{u}_{\lambda} \in C^{1}\left(\mathbb{R}^{N}\right)$ is a distribution solution of $P(\alpha, \beta, \lambda)$ on $\mathbb{R}^{N}$. Since $u_{\lambda}$ is a ground state, it is not hard to show that $u_{\lambda}$ has a connected support. Thus by Lemma 5.1, $\tilde{u}_{\lambda}$ is a radially symmetric function with respect to the centre of some ball $B_{R^{\lambda}}$ with a radius $R^{\lambda}>0$, so that $\operatorname{supp}\left(u_{\lambda}\right)=\bar{B}_{R^{\lambda}}$.

Let us show that $B_{R^{\lambda}}$ is an inscribed ball in $\Omega$. Consider $B_{\sigma R^{\lambda}}:=\left\{x \in \mathbb{R}^{N}: x / \sigma \in B_{R^{\lambda}}\right\}$ where $\sigma>0$. Notice that $B_{\sigma R^{\lambda}} \subset \Omega$ if $\sigma \leq 1$. Suppose, contrary to our claim, that there is $\sigma_{0}>1$ such that $B_{\sigma R^{\lambda}} \subset \Omega$ for any $\sigma \in\left(1, \sigma_{0}\right)$. Let $\sigma \in\left(1, \sigma_{0}\right)$. Introduce $u_{\lambda}^{\sigma}(x)=u_{\lambda}(x / \sigma), x \in B_{\sigma R^{\lambda}}$ and set $u_{\lambda}^{\sigma}(x)=0$ in $\Omega \backslash B_{\sigma R^{\lambda}}$. Observe that

$$
E_{\lambda}\left(u_{\lambda}^{\sigma}\right)=\frac{\sigma^{N-2}}{2} \int_{\Omega}\left|\nabla u_{\lambda}\right|^{2} \mathrm{~d} x-\sigma^{N}\left(\frac{\lambda}{\beta+1} \int_{\Omega}\left|u_{\lambda}\right|^{\beta+1} \mathrm{~d} x-\frac{1}{\alpha+1} \int_{\Omega}\left|u_{\lambda}\right|^{\alpha+1} \mathrm{~d} x\right) .
$$


From this $d E_{\lambda}\left(u_{\lambda}^{\sigma}\right) /\left.d \sigma\right|_{\sigma=1}=P_{\lambda}\left(u_{\lambda}\right)=0$, and thus $\sigma=1$ is a maximizing point of the function $\psi_{u}(\sigma):=E_{\lambda}\left(u_{\lambda}^{\sigma}\right)$. Then $E_{\lambda}\left(u_{\lambda}^{\sigma}\right)<E_{\lambda}\left(u_{\lambda}\right)=\hat{E}_{\lambda}$ and $P_{\lambda}\left(u_{\lambda}^{\sigma}\right)<0$ for $\sigma \in\left(1, \sigma_{0}\right)$. From this it follows that for $\sigma$ sufficiently close to 1 we have $E_{\lambda}\left(t_{\min }\left(u_{\lambda}^{\sigma}\right) u_{\lambda}^{\sigma}\right)<E_{\lambda}\left(u_{\lambda}\right)=\hat{E}_{\lambda}$ and $P_{\lambda}\left(t_{\min }\left(u_{\lambda}^{\sigma}\right) u_{\lambda}^{\sigma}\right)<0, E_{\lambda}^{\prime}\left(t_{\min }\left(u_{\lambda}^{\sigma}\right) u_{\lambda}^{\sigma}\right)=0$, which is a contradiction.

From this and Lemma 4.2 we have

Corollary 5.1. $u_{\lambda^{*}}$ is radially symmetric about some point of $\Omega$, and $\operatorname{supp}\left(u_{\lambda^{*}}\right)=\bar{B}_{R(\Omega)}$ is an inscribed ball in $\Omega$.

Furthermore, we have

Corollary 5.2. For any $\lambda>\lambda^{\star}$, problem $P(\alpha, \beta, \lambda)$ has no non-negative ground state with compact support.

Proof Suppose, conversely that there exists $\lambda_{a}>\lambda^{\star}$ and a ground state $u_{\lambda_{a}}$ of $P\left(\alpha, \beta, \lambda_{a}\right)$ such that $u_{\lambda_{a}}$ has a compact support. Then arguing as above one may infer that $u_{\lambda_{a}}$ is a radially symmetric function with respect to a centre of the inscribed ball $B_{R(\Omega)}$ in $\Omega$ so that $\operatorname{supp}\left(u_{\lambda_{a}}\right)=\bar{B}_{R(\Omega)}$. Consider $u_{\lambda^{*}}^{\sigma}(x)=u_{\lambda^{*}}(x / \sigma)$ with $\sigma=\left(\lambda^{\star} / \lambda_{a}\right)^{(1-\alpha) / 2(\beta-\alpha)}$. Then $u_{\lambda^{\star}}^{\sigma}$ is a compactly supported non-negative weak solution of $P\left(\alpha, \beta, \lambda_{a}\right)$. By the uniqueness of radial compact support solution of $P\left(\alpha, \beta, \lambda_{a}\right)$ (see Lemma 5.1) this is possible only if $u_{\lambda^{*}}^{\sigma}=u_{\lambda_{a}}$. However $\operatorname{supp}\left(u_{\lambda^{*}}^{\sigma}\right)=\bar{B}_{\sigma R(\Omega)}$ whereas $\operatorname{supp}\left(u_{\lambda_{a}}\right)=\bar{B}_{R(\Omega)}$ and $\sigma<1$. Thus we get a contradiction.

Corollary 5.3. $Z=\left(\lambda^{\star},+\infty\right)$.

Proof Suppose, contrary to our claim, that there is another limit point $\lambda_{b}$ of $Z$ such that $\lambda_{b} \in\left(\lambda^{\star},+\infty\right) \backslash Z$. Then arguing similarly to the proof of Lemma 4.2 one may conclude that there exists a compactly supported non-negative ground state $u_{\lambda_{b}}$ of $P\left(\alpha, \beta, \lambda_{b}\right)$. However $\lambda_{b}>\lambda^{*}$ and therefore by Corollary 5.2 this is impossible.

\section{Proofs of Theorems}

\subsection{Proof of Theorem 1.1}

For $\lambda=\lambda^{\star}$, the existence of non-negative ground state $u_{\lambda^{*}}$ of $P\left(\alpha, \beta, \lambda^{\star}\right)$ follows from Lemma 4.2. Since $Z=\left(\lambda^{\star},+\infty\right)$, we see that for $\lambda>\lambda^{\star}$, any minimizer $u_{\lambda}$ of (24) satisfies $P_{\lambda}\left(u_{\lambda}\right)<0$. From this by Proposition 4.2 we derive that $u_{\lambda}$ is a weak solution of $P(\alpha, \beta, \lambda)$. Moreover, since $\hat{E}_{\lambda}=E_{\lambda}\left(u_{\lambda}\right), u_{\lambda}$ is a ground state of $P(\alpha, \beta, \lambda)$ for all $\lambda \in\left(\lambda^{\star},+\infty\right)$. By the same arguments as in the proof of Lemma 4.2 we may assume that $u_{\lambda} \geq 0$ in $\Omega$ for all $\lambda>\lambda^{\star}$. In view of Lemma 2.2 we have $E_{\lambda}^{\prime \prime}\left(u_{\lambda}\right)>0$, and by global (up to the boundary) regularity results for elliptic equations we have $u_{\lambda} \in C^{1, y}(\bar{\Omega})$ for some $y \in(0,1)$.

Let us prove that for $\lambda<\lambda^{\star}$, problem $P(\alpha, \beta, \lambda)$ has no weak solution $u \in H_{0}^{1}(\Omega)$. Observe that any weak solution of $P(\alpha, \beta, \lambda)$ (if it exists) by global (up to the boundary) regularity results for elliptic equations belongs to $C^{1}(\bar{\Omega})$. Notice that by Corollary 3.3 for any $\lambda<\Lambda_{1 P}$ equation $P(\alpha, \beta, \lambda)$ has no weak solution $u \in C^{1}(\bar{\Omega})$. Thus since by Lemma 4.2, $\Lambda_{1 P}<\lambda^{\star}$ it remains to prove nonexistence of weak solutions in the case $\lambda \in\left[\Lambda_{1 P}, \lambda^{\star}\right)$.

Let $\lambda \in\left[\Lambda_{1 P}, \lambda^{*}\right)$. Suppose, contrary to our claim, that there exists a weak solution $u_{\lambda} \in C^{1}(\bar{\Omega})$ of $P(\alpha, \beta, \lambda)$. Then $E^{\prime}\left(u_{\lambda}\right)=0$ and by Corollary 2.1 we have $P_{\lambda}\left(u_{\lambda}\right) \leq 0$. Hence $u_{\lambda} \in M_{\lambda}$.

Let us show that then there exists a ground state of $P(\alpha, \beta, \lambda)$ which belongs to $C^{1}(\bar{\Omega})$. Notice that if $u_{\lambda}$ is a unique solution of $P(\alpha, \beta, \lambda)$ then it is a ground state. Assume there exists a set of such solutions $\tilde{M}_{\lambda}$ of $P(\alpha, \beta, \lambda)$. Notice that $\tilde{M}_{\lambda} \subset M_{\lambda}$. Consider

$$
\tilde{E}_{\lambda}:=\min _{u \in \tilde{M}_{\lambda}} E_{\lambda}(u) .
$$

Let $\left(u_{m}\right)$ be a minimizing sequence of (29), i.e.,

$$
E_{\lambda}\left(u_{m}\right) \rightarrow \tilde{E}_{\lambda} \text { as } n \rightarrow \infty \text { and } u_{m} \in \tilde{M}_{\lambda}, n=1,2, \ldots
$$


Using the same arguments as in the proof of Lemma 4.1 we may conclude that there exists a nonzero limit point $\tilde{u}_{0}$ such that (up to subsequence) $u_{m} \rightarrow \tilde{u}_{0}$ converges weakly in $H_{0}^{1}$ and strongly in $L_{q}$ for $1<q<2^{*}$. Then we have

$$
E_{\lambda}\left(\tilde{u}_{0}\right) \leq \tilde{E}_{\lambda}
$$

and

$$
0=D_{u} E_{\lambda}\left(u_{m}\right)(\psi) \rightarrow D_{u} E_{\lambda}\left(\tilde{u}_{0}\right)(\psi) \quad \forall \psi \in C_{0}^{\infty}(\Omega) .
$$

Thus $\tilde{u}_{0}$ is a nonzero weak solution of $P(\alpha, \beta, \lambda)$. Moreover by global (up to the boundary) regularity results for elliptic equations we have $\tilde{u}_{0} \in C^{1, y}(\bar{\Omega})$ for some $y \in(0,1)$. Thus $\tilde{u}_{0} \in \tilde{M}_{\lambda}$ and by (31) we conclude that $E_{\lambda}\left(\tilde{u}_{0}\right)=\tilde{E}_{\lambda}$. This implies that $\tilde{u}_{0}$ is a ground state of $P(\alpha, \beta, \lambda)$ belonging to $C^{1}(\bar{\Omega})$.

Thus we have proved that there exists a ground state $u_{\lambda}$ of $P(\alpha, \beta, \lambda)$ which belongs to $C^{1}(\bar{\Omega})$. Then there are two possibilities $P_{\lambda}\left(u_{\lambda}\right)<0$ or $P_{\lambda}\left(u_{\lambda}\right)=0$. In the first case, we get that $\lambda \in Z$. But in view of Corollary 5.3 this is a contradiction. In the second case, Corollary 2.1 implies that $u_{\lambda}$ has a compact support in $\Omega$. However the same arguments as in the proof of Corollary 5.2 show that for $\lambda \neq \lambda^{\star}$ this is impossible.

This concludes the proof of Theorem 1.1.

\subsection{Proof of Theorem 1.2}

The existence of a non-negative ground state $u_{\lambda^{*}}$ with compact support follows from Lemma 4.2 . By Corollary 5.1, $u_{\lambda^{*}}$ is radially symmetric about some point of $\Omega$, and $\operatorname{supp}\left(u_{\lambda^{*}}\right)=\bar{B}_{R(\Omega)}$ is an inscribed ball in $\Omega$.

In view of Corollary 5.2, for all $\lambda>\lambda^{\star}$, any ground state $u_{\lambda}$ of $P(\alpha, \beta, \lambda)$ is a "classical" non-negative ground state.

\subsection{Proof of Theorem 1.3}

We shall only prove the theorem, as an example, for the case $m=2$, i.e., when $\Omega$ is a domain of Strictly Starshaped Class 2.

Let $\lambda^{\star}>0$ be a limit value obtained in Theorem 1.1. By Lemma 4.2 there exists a compactly supported ground state $u_{\lambda^{\star}}^{1}$ of $P(\alpha, \beta, \lambda)$ and there exists a set of "classical" non-negative ground states $\left(u_{\lambda_{n}}^{1}\right)_{n=1}^{\infty}, \lambda_{n}>\lambda^{\star}$, $n=1,2, \ldots$ such that $u_{\lambda_{n}}^{1} \rightarrow u_{\lambda^{\star}}^{1}$ strongly in $H_{0}^{1}$ as $n \rightarrow \infty$. By Corollary 5.1, $u_{\lambda^{\star}}$ is radially symmetric about some origin $0 \in \Omega$, and $\operatorname{supp}(u)=\bar{B}_{R(\Omega)}$ is an inscribed ball in $\Omega$. By the assumptions $\Omega$ contains exactly 2 inscribed balls of radius $R(\Omega)$

Set $u_{\lambda^{*}}^{2}(x):=u_{\lambda^{*}}^{1}\left(R_{H} x\right), u_{\lambda_{n}}^{2}(x):=u_{\lambda_{n}}^{1}\left(R_{H} x\right), x \in \Omega, n=1,2, \ldots$, where $R_{H}: \mathbb{R}^{N} \rightarrow \mathbb{R}^{N}$ is the reflection map. By Theorem 1.1, the support of $u_{\lambda^{*}}^{1}$ coincides with one of the balls $B^{1}$ or $B^{2}$. Assume $\operatorname{supp}\left(u_{\lambda^{*}}^{1}\right)=B^{1}$. Then since $R_{H} B_{1}=B_{2}$ for some hyperplane $H$, we have $\operatorname{supp}\left(u_{\lambda^{*}}^{2}\right)=B^{2}$ and thus $u_{\lambda^{*}}^{2} \neq u_{\lambda^{*}}^{1}$. Since $u_{\lambda_{n}}^{2} \rightarrow u_{\lambda^{*}}^{2}$ strongly in $H_{0}^{1}$ as $n \rightarrow \infty$, it follows that $u_{\lambda_{n}}^{1} \neq u_{\lambda_{n}}^{2}$ for sufficiently large $n$.

\section{On the quasilinear parabolic problem}

In this last section part we consider the associate quasilinear parabolic problem of porous media type

$$
P P\left(m, \alpha, \beta, \lambda, v_{0}\right) \begin{cases}\left(|v|^{\frac{1}{m}-1} v\right)_{t}-\Delta v+|v|^{\alpha-1} v=\lambda|v|^{\beta-1} v & \text { in }(0,+\infty) \times \Omega \\ v=0 & \text { on }(0,+\infty) \times \partial \Omega \\ v(0, x)=v_{0}(x) & \text { on } \Omega,\end{cases}
$$


where $m>0$ and always under the structural assumption $0<\alpha<\beta<1$. As mentioned before, when $m \neq 1$ the problem usually appears in the literature formulated, equivalently, in terms of $w:=|v|^{\frac{1}{m}-1} v$

$$
\overline{P P}\left(m, a, b, \lambda, w_{0}\right) \quad \begin{cases}w_{t}-\Delta|w|^{m-1} w+|w|^{a-1} w=\lambda|w|^{b-1} w & \text { in }(0,+\infty) \times \Omega \\ w=0 & \text { on }(0,+\infty) \times \partial \Omega \\ w(0, x)=w_{0}(x) & \text { on } \Omega,\end{cases}
$$

with $a=\alpha m, b=\beta m$ and $w_{0}:=\left|v_{0}\right|^{\frac{1}{m}-1} v_{0}$. The parabolic semilinear case $m=1$ was treated in the previous paper by the authors [11] and many other references where collected there. For the basic theory for this problem, always under the structural assumption $0<a<b<m$, we send the reader to [11, 31] (for the case $m=1$ ) and to [32] (for the case $m \neq 1$ ). We notice that some of the results presented in [11] does not appear explicitely written in [32] but its adaptation to the quasilinear framework are today standard (see also [2224]). So, in particular, we know that for any $v_{0} \in \mathrm{L}^{\infty}(\Omega), v_{0} \geq 0$ there exists a nonnegative weak solution of $P P\left(m, \alpha, \beta, \lambda, v_{0}\right)$ with $w \in \mathcal{C}\left([0,+\infty), \mathrm{L}^{1}(\Omega)\right) \cap L^{\infty}((0,+\infty) \times \Omega), v \in L^{\infty}((0,+\infty) \times \Omega) \cap L_{l o c}^{2}\left(\delta, T: H_{0}^{1}(\Omega)\right)$, for any $0<\delta<T$. This solution is unique if $v_{0}$ is non-degenerate near its free boundary. The two next subsections collect our results concerning the parabolic problem $P P\left(m, \alpha, \beta, \lambda, v_{0}\right)$ under two different points of view.

\subsection{On the stability of flat solution}

Our main goal here is to prove the following result extending Theorem 1.1, part (2) of [11] (concerning $\left.\lambda=\lambda^{\star}\right)$ to the case $m \neq 1$.

Theorem 7.1. Assume $0<a<b<m$ such that

$$
2(m+a)(m+b)-N(m-a)(m-b)<0,
$$

then, if $\alpha=a / m$ and $\beta=b / m$, the stationary ground state $u_{\lambda^{*}} \in H_{0}^{1}(\Omega)$ of problem $P\left(\alpha, \beta, \lambda^{\star}\right)$ is a $H_{0}^{1}$-stable solution of $\overline{P P}\left(m, a, b, \lambda^{\star}, w_{0}\right)$, i.e., given any $\varepsilon>0$, there exists $\delta>0$ such that

$$
\left\|u_{\lambda^{*}}-|w|^{m-1} w\left(t ; w_{0}\right)\right\|_{1}<\varepsilon \text { for any } w_{0} \text { s.t. }\left\|u_{\lambda^{*}}-\left|w_{0}\right|^{m-1} w_{0}\right\|_{1}<\delta, \quad \forall t>0,
$$

where $w\left(t ; w_{0}\right)$ is the weak solution of $\overline{P P}\left(m, a, b, \lambda^{\star}, w_{0}\right)$.

Proof. As in the proof of Theorem 1.1, part (2) of [11] there are two different kinds of arguments. On one hand, we first prove that under the assumption (34) the ground state $u_{\lambda^{*}}$ is $H_{0}^{1}(\Omega)$-isolated. Indeed, it is enough to use the uniqueness of the compact supported solution and that from the proof of Theorem 1.3. we know that there exists a set of "classical" non-negative ground states $\left(u_{\lambda_{n}}^{1}\right)_{n=1}^{\infty}, \lambda_{n}>\lambda^{\star}, n=1,2, \ldots$ such that $u_{\lambda_{n}}^{1} \rightarrow u_{\lambda^{*}}^{1}$ strongly in $H_{0}^{1}(\Omega)$ as $n \rightarrow \infty$. The second ingredient of the proof consists in showing that the energy functional $E_{\lambda}(u)$ (here $\lambda>0$ is arbitrary) is a Lyapounov function in the sense that

$$
\frac{\partial}{\partial t} E_{\lambda}(v(t)) \leq 0 \text { in }(0, T)
$$

for any $T>0$ and for any $v(t)$ weak solution of $P P\left(m, \alpha, \beta, \lambda, v_{0}\right)$. In the semilinear case, $m=1$, that was proved in Lemma 6.2 of [11]. The case $m \neq 1$ requires a slight modification since the control of the time derivative of $v(t)$ is more delicate. Nevertheless, it is easy to adapt the regularity results of [22] (see also [24, 33]) to prove that if $v(t)$ is a weak solution of $P P\left(m, \alpha, \beta, \lambda, v_{0}\right)$ with a smooth initial datum then $\left(|v(t)|^{\frac{1}{m}-1} v(t)\right)_{t} \in$ $L^{2}(\Omega)$ for $t>0$. Then we can use well-known regularizing arguments (so that $v_{t}(t) \in L^{2}(\Omega)$ ) and then passing to the limit to get, as in the Appendix of [11], that

$$
\begin{aligned}
\frac{\partial}{\partial t} E_{\lambda}(v(t)) & =D_{u} E_{\lambda}(v(t))\left(v_{t}(t)\right)= \\
& <-\Delta v(t)-\lambda|v|^{\beta-1} v+|v|^{\alpha-1} v, v_{t}(t)>= \\
-\frac{1}{m \omega^{2}} \int_{\Omega}\left|\left(v^{\omega}(t)\right)_{t}\right|^{2} d x & \leq 0
\end{aligned}
$$


with $\omega=(1+m) / 2 m$ and thus we get the result.

\subsection{On the free boundary}

Our main goal in this Section is to give an idea of the time evolution of the support of the solution. We recall that, as $t \rightarrow+\infty$, the support of $v\left(t\right.$. .) must converge to a ball of $\mathbb{R}^{N}$, in the case $\lambda=\lambda^{\star}$, or to the whole domain $\bar{\Omega}$, if $\lambda>\lambda^{\star}$ (since the shape of the support of the associated stationary solutions was given in Theorem 1.1).

Our first result concerns the special case of $v_{0}=u_{\lambda^{*}}$ (i.e. with support in the ball of $\mathbb{R}^{N}$ of radius $R(\Omega)$ ) and $\lambda>\lambda^{\star}$. It is clear that any stationary solution $u_{\lambda^{*}}$ is a subsolution to the problem $P P\left(m, \alpha, \beta, \lambda, v_{0}\right)$. Indeed,

$$
\left(\left|u_{\lambda^{\star}}\right|^{\frac{1}{m}-1} u_{\lambda^{\star}}\right)_{t}-\Delta u_{\lambda^{\star}}+\left|u_{\lambda^{\star}}\right|^{\alpha-1} u_{\lambda^{\star}}=\lambda^{\star}\left|u_{\lambda^{\star}}\right|^{\beta-1} u_{\lambda^{\star}}<\lambda\left|u_{\lambda^{\star}}\right|^{\beta-1} u_{\lambda^{\star}} .
$$

So, if $u_{\lambda^{\star}}$ is nondegenerate near its free boundary, we get that $u_{\lambda^{*}}(x) \leq v(t, x)$ for any $t>0$ and a.e. $x \in \Omega$. As a matter of fact, it is easy to prove that under these assumptions $v_{t} \geq 0$ a.e. $(0,+\infty) \times \Omega$. Thus, a priori, the support of the solution $v(t,$.$) is greater or equal to the support of u_{\lambda^{*}}$ for any $t>0$. The following result gives some indication about how the support of $v(t,$.$) should increase slowly with time. We shall apply the general$ local energy methods for the study of free boundary problems (see, e.g. [34]). Notice that for our goal we only need to get some information on $v(t$, . $)$ on the level sets where this function is small enough. So, given $\theta>0$ and $t \geq 0$ we introduce the notation

$$
\Omega_{v, \theta}(t):=\{x \in \Omega: v(t, x) \leq \theta\} .
$$

Theorem 7.2. Assume $0<a<b<m, \lambda>\lambda^{\star}, v_{0}=u_{\lambda^{\star}}$ and let $\theta>0$ such that $\theta^{\beta-\alpha}<1 / \lambda$. Let $x_{0} \in \mathbb{R}^{N} \backslash \operatorname{supp}\left(v_{0}\right)$ such that $B_{\rho_{0}}\left(x_{0}\right) \subset \mathbb{R}^{N} \backslash \operatorname{supp}\left(v_{0}\right)$ for some $\rho_{0}>0$. Then there exists $\widehat{t}>0$ and a continuous decreasing function $\rho:[0, \widehat{t}] \rightarrow\left[0, \rho_{0}\right]$ such that $\rho(0)=\rho_{0}, \rho(\widehat{t})=0$ and $B_{\rho(t)}\left(x_{0}\right) \subset\left(\mathbb{R}^{N} \backslash \operatorname{supp}(v(t,).) \cap \Omega_{v, \theta}(t)\right.$ for any $t \in[0, \widehat{t}]$. In particular, $v(t, x)=0$ a.e. $x \in B_{\rho(t)}\left(x_{0}\right)$ for any $t \in[0, \widehat{t}]$.

Proof It is enough to apply Theorem 2.2 of [34] to the special case of $\psi(u)=|u|^{\frac{1}{m}-1} u$ and

$$
A(x, t, u, D u)=D u, B(x, t, u, D u)=0, C(x, t, u, D u)=\left(1-\lambda \theta^{\beta-\alpha}\right)|u|^{\alpha-1} u,
$$

since we know that

$$
\left(|v|^{\frac{1}{m}-1} v\right)_{t}-\Delta v+\left(1-\lambda \theta^{\beta-\alpha}\right)|v|^{\alpha-1} v \leq 0 \text { on } \cup_{t>0}\{t\} \times \Omega_{v, \theta}(t),
$$

and all the assumptions of Theorem 2.2 of [34] hold.

When $\lambda=\lambda^{\star}$ we can also give an idea how the support of $v(t,$.$) corresponding to a strictly positive ini-$ tial datum decreases, after a finite time large enough (remember that in that case the support of $v(t,$.$) must$ decrease from $\bar{\Omega}$ to the closed ball of $\mathbb{R}^{N}$ of radius $R(\Omega)$ contained in $\Omega$ ). In this case, we shall pay attention to the special choice of $v_{0}=u_{\lambda}$ for some $\lambda>\lambda^{\star}$. Notice that now $u_{\lambda}$ is a supersolution to $P P\left(m, \alpha, \beta, \lambda^{\star}, v_{0}\right)$ since

$$
\left(\left|u_{\lambda}\right|^{\frac{1}{m}-1} u_{\lambda}\right)_{t}-\Delta u_{\lambda}+\left|u_{\lambda}\right|^{\alpha-1} u_{\lambda}=\lambda\left|u_{\lambda}\right|^{\beta-1} u_{\lambda}>\lambda^{\star}\left|u_{\lambda^{\star}}\right|^{\beta-1} u_{\lambda^{\star}} .
$$

As above, if $u_{\lambda}$ is nondegenerate, we can even prove that $v_{t} \leq 0$ a.e. $(0,+\infty) \times \Omega$. Concerning the formation of the free boundary we have:

Theorem 7.3. Assume $0<a<b<m, \lambda=\lambda^{\star}, v_{0}=u_{\lambda}$ for some $\lambda>\lambda^{\star}$ and let $\theta>0$ such that $\theta^{\beta-\alpha}<1 / \lambda^{\star}$. Then, for any time $T>0$ large enough, there exist a finite time $t^{\#}>0$ and a continuous increasing function $\rho:\left[t^{\#}, T\right] \rightarrow[0,+\infty)$ such that $\rho\left(t^{\#}\right)=0$, and $B_{\rho_{0}}\left(x_{0}\right) \subset\left(\mathbb{R}^{N} \backslash \operatorname{supp}(v(t,).) \cap \Omega_{v, \theta}(t)\right.$ for any $t \in\left[t^{\#}, T\right]$. In particular, $v(t, x)=0$ a.e. $x \in B_{\rho(t)}\left(x_{0}\right)$ for any $t \in\left[t^{\#}, T\right]$. 
Proof This time it is enough to apply Theorem 4.2 of [34] to the special case of $\psi(u)=|u|^{\frac{1}{m}-1} u$, $A(x, t, u, D u)=D u, B(x, t, u, D u)=0$ and

$$
C(x, t, u, D u)=\left(1-\lambda^{\star} \theta^{\beta-\alpha}\right)|u|^{\alpha-1} u .
$$

Indeed, as above we know that

$$
\left(|v|^{\frac{1}{m}-1} v\right)_{t}-\Delta v+\left(1-\lambda^{\star} \theta^{\beta-\alpha}\right)|v|^{\alpha-1} v \leq 0 \text { on } \cup_{t \in(0, T)}\{t\} \times \Omega_{v, \theta}(t) .
$$

and all the assumptions of Theorem 4.2 of [34] hold.

\section{A Appendix}

Lemma A.1. Assume $\lambda \in\left[\Lambda_{1 P},+\infty\right)$ and $u_{\lambda_{m}}$ is a sequence of solutions of (24), where $\lambda_{m} \rightarrow \lambda$ as $m \rightarrow+\infty$. Then there exist a minimizer $u_{\lambda}$ of (24) and a subsequence, still denoted by $\left(u_{\lambda_{m}}\right)$, such that $u_{\lambda_{m}} \rightarrow u_{\lambda}$ strongly in $H_{0}^{1}$ as $m \rightarrow+\infty$.

Proof Let $\lambda \in\left[\Lambda_{1 P},+\infty\right), \lambda_{m} \rightarrow \lambda$ as $m \rightarrow+\infty$ and $u_{\lambda_{m}}$ be a sequence of solutions of (24). As in the proof of Lemma 4.1 it is derived that the set $\left(u_{\lambda_{m}}\right)$ is bounded in $H_{0}^{1}$. Hence by the Sobolev embedding theorem there exists a subsequence, still denoted by $\left(u_{\lambda_{m}}\right)$, such that

$$
u_{\lambda_{m}} \rightarrow \bar{u}_{\lambda} \text { weakly in } H_{0}^{1}, \quad u_{\lambda_{m}} \rightarrow \bar{u}_{\lambda} \text { strongly in } L^{q}(\Omega),
$$

where $0<q<2^{*}$, for some limit point $\bar{u}_{\lambda}$. As in the proof of Lemma 4.1 one derives that $\bar{u}_{\lambda} \neq 0$ and

$$
E_{\lambda}\left(\bar{u}_{\lambda}\right) \leq \liminf _{m \rightarrow \infty} E_{\lambda_{m}}\left(u_{\lambda_{m}}\right), \quad E_{\lambda}^{\prime}\left(\bar{u}_{\lambda}\right) \leq 0, \quad P_{\lambda}\left(\bar{u}_{\lambda}\right) \leq 0 .
$$

Let $\lambda>\Lambda_{1 P}$. By Lemma 4.1 there exists a minimizer $u_{\lambda}$ of (24), i.e. $u_{\lambda} \in M_{\lambda}$ and $\hat{E}_{\lambda}=E_{\lambda}\left(u_{\lambda}\right)$. Then

$$
\left|E_{\lambda}\left(u_{\lambda}\right)-E_{\lambda_{m}}\left(u_{\lambda}\right)\right|<C\left|\lambda-\lambda_{m}\right|,
$$

where $C<+\infty$ does not depend on $m$. Furthermore,

$$
E_{\lambda_{m}}\left(u_{\lambda}\right) \geq E_{\lambda_{m}}\left(t_{\min }\left(u_{\lambda}\right) u_{\lambda}\right) \geq E_{\lambda_{m}}\left(u_{\lambda_{m}}\right)
$$

provided that $m$ is a sufficiently large number. Thus we have

$$
E_{\lambda}\left(u_{\lambda}\right)+C\left|\lambda-\lambda_{m}\right|>E_{\lambda_{m}}\left(u_{\lambda}\right) \geq E_{\lambda_{m}}\left(u_{\lambda_{m}}\right),
$$

and therefore $\hat{E}_{\lambda}:=E_{\lambda}\left(u_{\lambda}\right) \geq \liminf _{m \rightarrow \infty} E_{\lambda_{m}}\left(u_{\lambda_{m}}\right)$. Hence by (38) we have

$$
E_{\lambda}\left(\bar{u}_{\lambda}\right) \leq \hat{E}_{\lambda} \text {. }
$$

Assume $E_{\lambda}^{\prime}\left(\bar{u}_{\lambda}\right)<0$. Then $E_{\lambda}^{\prime}\left(t_{\min }\left(\bar{u}_{\lambda}\right) \bar{u}_{\lambda}\right)=0$ and $E_{\lambda}\left(t_{\min }\left(\bar{u}_{\lambda}\right) \bar{u}_{\lambda}\right)<E_{\lambda}\left(\bar{u}_{\lambda}\right) \leq \hat{E}_{\lambda}$. In virtue of Proposition 2.1, this implies that $P_{\lambda}\left(t_{\min }\left(\bar{u}_{\lambda}\right) \bar{u}_{\lambda}\right)<0$. Thus $t_{\min }\left(\bar{u}_{\lambda}\right) \bar{u}_{\lambda} \in M_{\lambda}$ and since $E_{\lambda}\left(t_{\min }\left(\bar{u}_{\lambda}\right) \bar{u}_{\lambda}\right)<\hat{E}_{\lambda}$ we get a contradiction. Hence $E_{\lambda}\left(\bar{u}_{\lambda}\right)=\hat{E}_{\lambda}, E_{\lambda}^{\prime}\left(\bar{u}_{\lambda}\right)=0$ and $u_{\lambda_{m}} \rightarrow \bar{u}_{\lambda}$ strongly in $H_{0}^{1}$ as $m \rightarrow+\infty$.

Assume now that $\lambda=\Lambda_{1 P}$. Since $E_{\lambda_{m}}^{\prime}\left(u_{\lambda_{m}}\right)=0, P_{\lambda_{m}}\left(u_{\lambda_{m}}\right) \leq 0$, we have $r_{u_{\lambda_{m}}}^{P}(1) \leq \lambda_{m}=r_{u_{\lambda_{m}}}^{1}(1)$. Then by Proposition 3.4 (see Figure 5), $1 \in\left[t_{1 P}\left(u_{\lambda_{m}}\right),+\infty\right)$ and therefore

$$
\lambda_{1 P}\left(u_{\lambda_{m}}\right)=r_{u_{\lambda_{m}}}^{1}\left(t_{1 P}\left(u_{\lambda_{m}}\right)\right) \leq r_{u_{\lambda_{m}}}^{1}(1)=\lambda_{m}, \quad m=1,2, \ldots .
$$

Hence, since $\lambda_{m} \downarrow \lambda$, we have $\lambda_{1 P}\left(u_{\lambda_{m}}\right) \downarrow \Lambda_{1 P}$ as $m \rightarrow \infty$. Thus, $\left(u_{\lambda_{m}}\right)$ is a minimizing sequence of (22) and therefore by (37), $\lambda_{1 P}\left(\bar{u}_{\lambda}\right) \leq \Lambda_{1 P}$. Since the strict inequality $\lambda_{1 P}\left(\bar{u}_{\lambda}\right)<\Lambda_{1 P}$ is impossible, we conclude that $\lambda_{1 P}\left(\bar{u}_{\lambda}\right)=\Lambda_{1 P}$, which yields that $u_{\lambda_{m}} \rightarrow \bar{u}_{\lambda}$ strongly in $H_{0}^{1}$.

\section{Acknowledgments}

The research of J.I. Díaz and J. Hernández was partially supported by the projects ref. MTM 2014-57113-P and MTM2017-85449-P of the DGISPI (Spain). 


\section{References}

[1] P. Pucci and J. Serrin, Uniqueness of ground states for quasilinear elliptic operators. Indiana University Mathematics Journal 47 (1998) 2501-528.

[2] F. Gazzola, J. Serrin and M. Tang, Existence of ground states and free boundary problems for quasilinear elliptic operators, Advances in Differential Equations 5 (2000) 1-30.

[3] J. Serrin and H. Zou, Symmetry of ground states of quasilinear elliptic equations. Archive for Rational Mechanics and Analysis, 148 (1999) 4 265-290.

[4] J.I. Díaz and J. Hernández,, Global bifurcation and continua of nonnegative solutions for a quasilinear elliptic problem, C.R. Acad. Sci. Paris, 329, (1999), 587-592.

[5] J. I. Díaz, J. Hernández and F. J. Mancebo, Branches of positive and free boundary solutions for some singular quasilinear elliptic problems, J. Math. Anal. Appl., 352 (2009), 449-474.

[6] C. Cortázar, M. Elgueta, and P. Felmer, Symmetry in an elliptic problem and the blow-up set of a quasilinear heat equation, Comm. P.D.E., 21 (1996) 507-520.

[7] C. Cortázar, M. Elgueta, and P. Felmer, On a semi-linear elliptic problem in $\mathbb{R}^{N}$ with a non-Lipschitzian non-linearity, $A d$ vances in Diff. Eqs., 1 (1996) 199-218.

[8] H. Kaper and M. Kwong, Free boundary problems for Emden-Fowler equation, Differential and Integral Equations, 3 (1990) 353-362.

[9] H. Kaper, M. Kwong and Y. Li, Symmetry results for reaction-diffusion equations, Differential and Integral Equations, 6 (1993) 1045-1056.

[10] J. I. Díaz, J. Hernández and Y. Sh. Il'yasov, On the existence of positive solutions and solutions with compact support for a spectral nonlinear elliptic problem with strong absorption, Nonl. Anal.: Th., Meth. \& Appl. 119 (2015), 484-500.

[11] J. I. Díaz, J. Hernández and Y. Sh. Il'yasov, Stability criteria on flat and compactly supported ground states of some nonLipschitz autonomous semilinear equations, Chinese Ann. Math. 38 (2017), 345-378.

[12] Y. Sh. Il'yasov, On the curve of critical exponents for nonlinear elliptic problems in the case of a zero mass. Computational Mathematics and Mathematical Physics 57 (2017), 3 497-514.

[13] P. Rosenau and J. M. Hyman, Compactons: Solitons with finite wavelength, Phys. Rev. Lett. 70 (1993) 5 564-567.

[14] Y. Sh. Ilyasov and Y. Egorov, Hopf maximum principle violation for elliptic equations with non-Lipschitz nonlinearity, Nonlin. Anal. 72 (2010) 3346-3355.

[15] Y. Sh. Il'yasov, On critical exponent for an elliptic equation with non-Lipschitz nonlinearity, Dynamical Systems, Supplement (2011), 698-706.

[16] J. Hernández, F. Mancebo and J. M. Vega, Positive solutions for singular nonlinear elliptic equations, Proc. Roy. Soc. Edinburgh 137A (2007), 41-62.

[17] M. Montenegro and E. Silva, Two solutions for a singular elliptic equation by variational methods. Ann. Sc. Norm. Sup. Pisa 11 (2012), 143-165.

[18] A. Anello and F. Faraci, Two solutions for an elliptic problem with two singular terms. Calc. Var. and PDEs 56 (2017), 56-91.

[19] H. Fujita, On the blowing-up of solutions of the Cauchy problem for $u_{t}=\Delta u+u^{1+\alpha}$. J. Fac. Sci.Univ. Tokyo Sec. IA Math. 16 (1966) 105-113.

[20] A. A. Samarskii, V. A. Galaktionov, S. P. Kurdyumov, A. P. Mikhailov, Blow-up in quasilinear parabolic equations, Nauka, Moscow, 1987; English translation: Walter de Gruyter, Berlin/New York, 1995.

[21] V.A. Galaktionov and J.L. Vázquez. A Stability Technique for Evolution Partial Differential Equations. A Dynamical Systems Approach. Progress in Non-Linear Differential Equations and Their Applications 56, Birkhäuser Verlag, 2003.

[22] M. Efendiev and S. Zelik, Finite- and infinite-dimensional attractors for porous media equations. Proceedings of the London Mathematical Society 96 (2008) 51-77.

[23] M. Efendiev, Infinite dimensional attractors for porous medium equations in heterogeneous medium. Mathematical Methods in the Applied Sciences 35 (2012) 1987-1996.

[24] W. Niu, On the dimension of global attractors for porous media equations, Mathematical Methods in the Applied Sciences 36 (2013) 2588-2592.

[25] Y. Sh. Il'yasov and P. Takac, Optimal-regularity, Pohozhaev's identity, and nonexistence of weak solutions to some quasilinear elliptic equations. Journal of Differential Equations, 252 (2012) 3 2792-2822.

[26] S.I. Pohozaev, Eigenfunctions of the equation $\Delta u+\lambda f(u)=0$, Sov. Math. Doklady 5 (1965) 1408-1411.

[27] R. Temam, Navier-Stokes Equations: Theory and Numerical Analysis, North-Holland, Amsterdam-New York-0xford, 1979.

[28] Y. Sh. Ilyasov, On extreme values of Nehari manifold method via nonlinear Rayleigh's quotient. Topological Methods in Nonlinear Analysis 49 (2017) 2 683-714.

[29] E. Zeidler, Nonlinear functional analysis, Vol.3. Variational methods and optimization, Springer-Verlag, Berlin, 1985.

[30] G. M. Lieberman, Boundary regularity for solutions of degenerate elliptic equations, Nonlinear Anal., 12 (1988) $111203-$ 1219.

[31] T. Cazenave, T. Dickstein and M. Escobedo, A semilinear heat equation with concave-convex nonlinearity, Rendiconti di Matematica, Serie VII, 19 (1999) 211-242. 
[32] J. L. Vázquez, The porous medium equation. Mathematical theory. Oxford Mathematical Monographs. The Clarendon Press Oxford University Press, Oxford, 2007.

[33] Ph. Bénilan, Sur un problème d'évolution non monotone dans $L^{2}(\Omega)$, Publications Mathématiques de la Faculté des Sciences de Besançon. Fascicule $\mathrm{n}^{\circ} 2$ (1975-76).

[34] A.S. Antontsev, J. I. Díaz, S. Shmarev, Energy methods for free boundary problems. Applications to nonlinear PDEs and Fluid Mechanics, Birkäuser, Boston, 2002. 